\title{
BOUNDARY PROPERTIES OF SOLUTIONS OF DIFFERENTIAL EQUATIONS AND GENERAL BOUNDARY-VALUE PROBLEMS
}

\author{
V. P. BURSKII
}

\begin{abstract}
For a general differential operator with smooth matrix-valued coefficients in a bounded domain with smooth boundary we consider the boundary properties of functions from the domain of definition of a maximal extension in $L_{2}(\Omega)$ and we study the properties of extensions and boundary-value problems corresponding to them. The investigations are based on Green's formula.
\end{abstract}

\section{INTRODUCTION}

For many years the theory of boundary-value problems for partial differential equations was developed in terms of concrete boundary-value problems, and the passage from the particular to the general stayed within the confines determined by the fact that the theory was divided according to the type of equation. The foundations of the general theory of boundary-value problems, irrespective of the type of equation, were laid in the celebrated work of Vishik [18, in which the boundary-value problem appears in the specification of the domain of definition of some extension of a minimal operator. From this point of view, adopted in the theory of selfadjoint ordinary differential operators following von Neumann [34, one would probably view the papers of Calkin [48] and M.G. Kreln [25] as predecessors of [18. The next important step in the development of the theory, due to Hörmander in his paper [45, was to introduce the notion of a Cauchy space and to refine the notion of a boundary-value problem together with the interpretation of the results of [18, and the proof of the Vishik conditions for scalar differential operators with constant coefficients. There was a boom in interest in this topic in the 1960s, but the interest of analysts fell away after its difficulties were recognised, not the least that there had been no serious progress made. Among the works from the 1960s we note the investigations by Berezanskiu (see [2]) on smoothly generated general boundary-value problems, Agranovich [1] and Palamodov [35] on operators with constant coefficients. We also draw attention to the much later work due to Dezin 23, which was directed towards the analysis and classification of the most simply posed boundary-value problems, and the approximation of $L_{2}$-solutions by smooth functions in the graph norm. After the papers of Grubb [51, Rofe-Beketov [41] and Gorbachuk 21] a study of an abstract analogue of Hörmander's Cauchy space began. This is the notion of a space of boundary values, the first and most striking example of which had already been given for the case of several variables by Vishik in [18. The first case to be studied was the symmetric case, which was investigated in the papers of Kochuber [27, Lyantse and Storozh [31, Malamud and Derkach [53] (for symmetric linear relations see the paper by Malamud and Mogilevskii [54]), then for the more general case by Vaunnerman [17] (see the survey [20]). We note the papers on boundary values of solutions by Gorbachuk and Gorbachuk [19] and Roitberg [38, 39], and the works of Lyantse and Storozh on the description of

2000 Mathematics Subject Classification. Primary 35G05, 35B30, 35E20, 35A05, 35B05. 
the action of maximal operators and perturbations [31. There is a whole collection of papers on the properties of classes of operators, on the boundary behaviour of solutions, on the comparison of minimal and maximal operators, on equations with operator-valued coefficients and other closely related questions. A complete list would occupy too much space.

Boundary-value problems for differential equations in bounded domains for equations that are not elliptic have been systematically studied, mainly in a parallelepiped. In the plane, the uniqueness of the solution of the Dirichlet problem for second-order hyperbolic equations on domains with a generic boundary has been studied (see the survey [37]). For other possible topics in the study of boundary-value problems, in particular, when condition (2.15) holds, see [4, 6, 7, 8, 12, 13.

This paper is concerned with general questions in the theory of boundary-value problems for the case when a general Green's formula is available. In $\S 0$ the reader will find the notation and main properties of the spaces and operators used in what follows. In $\S 1$ we present the main definitions and well-known facts, as well as certain other matters. In particular, one consequence of this discussion is a proof of the well-posedness of a nonclassical boundary-value problem for the wave equation in a disc. In $\S 2$ we introduce and study the so-called $L$-traces of elements in the domain of definition of a maximal operator, which are responsible for the boundary properties of these elements. Their existence follows from Green's formula. In $\S 3$ we use the example of a general scalar differential operator and, in connection with the $L$-traces, we answer questions about the structure of the domain of definition of a minimal operator, the existence of a well-posed boundary-value problem, the comparison of minimal operators with variable coefficients, the existence of fundamental solutions, the representation of solutions as analogues of classical potentials and the validity of mean-value theorems. In $\S 4$ we consider a realization of a boundary space, going back to von Neumann, in which Green's formula takes on a simple form; we also describe a certain algorithm for the solution of a general well-posed boundary-value problem based on the solution of the generalized Dirichlet problem for an equation with a selfadjoint operator of divergence form. Section 5 is devoted to some illustrations of the above concepts, and in $\S 6$ we discuss our ideas for possible further developments in the theory. The final section $(\S 7)$ consists of comments and remarks and some references to papers we did not include in the main text. This paper is a continuation of the author's earlier research, which can be found in [5, 11, 15, 16].

\section{Notation AND initial ELEMENTs of The CONSTRUCTION 1}

In this section, for the convenience of the reader, we give the notation for spaces and operators in a unified form. We also state their main properties and certain assumptions that will be used in this paper; here the material in subsection I relates to $\S 1$ and $\S 4$, while that in subsections I and II relates to $\S \S 2,3,5$ and 6 .

I. $\left.I_{1}\right)$. Let $\Omega$ be a domain in $\mathbb{R}^{n}$, which for simplicity we assume to be bounded with an $(n-1)$-dimensional boundary $\partial \Omega$ on one side of it. For ease of notation we shall write $H:=L_{2}^{N}(\Omega)=\left[L_{2}(\Omega)\right]^{N}, H_{0}^{l}:=\left[H_{0}^{l}(\Omega)\right]^{N}, H^{l}:=\left[H^{l}(\Omega)\right]^{N}, H^{+}:=\left[L_{2}(\Omega)\right]^{N^{+}}$, $H_{0}^{+l}:=\left[H_{0}^{l}(\Omega)\right]^{N^{+}}, H^{+l}:=\left[H^{l}(\Omega)\right]^{N^{+}}\left(l, N, N^{+} \in \mathbb{N}\right)$, which are Sobolev spaces; $H_{0}^{l} \subset$ $H^{l}, H_{0}^{+l} \subset H^{+l}$ are embeddings of closed subspaces.

\footnotetext{
${ }^{1}$ An abstract scheme and the construction of the theory based on it is realized in the author's book [13.
} 
$\left.\mathrm{I}_{2}\right)$. Let $\mathcal{L}: H^{l} \rightarrow H^{+}$, that is, $\mathcal{L}:\left[H^{l}(\Omega)\right]^{N} \rightarrow\left[L_{2}(\Omega)\right]^{N^{+}}$, be a general differential operator without type:

$$
\mathcal{L}=\sum_{|\alpha| \leq l} a_{\alpha}(x) D^{\alpha}, \quad a_{\alpha} \in C^{\infty}(\bar{\Omega}), \quad D^{\alpha}=\frac{(-i \partial)^{|\alpha|}}{\partial x^{\alpha}}
$$

where the $a_{\alpha}(x)$ are $\left(N^{+} \times N\right)$-matrix coefficients whose entries are $C^{\infty}(\bar{\Omega})$-smooth complex-valued functions in the domain $\Omega$.

Suppose also that $\mathcal{L}^{+}: H^{+l} \rightarrow H$ is its formal adjoint operator:

$$
\mathcal{L}^{+}=\sum_{|\alpha| \leq l} D^{\alpha}\left(a_{\alpha}^{*}(x) \cdot\right)
$$

where $a_{\alpha}^{*}(x)$ is the adjoint matrix.

The operators $\mathcal{L}, \mathcal{L}^{+}$, called operations in what follows, are related by the formula

$$
(\mathcal{L} u, v)_{H^{+}}=\left(u, \mathcal{L}^{+} v\right)_{H},
$$

$u \in H^{l}, v \in H^{+l}$, where at least one of $u$ or $v$ is compactly supported, that is, $u \in H_{0}^{l}$ or $v \in H_{0}^{+l}$.

II. Supplement to I.

$\left.\mathrm{II}_{0}\right)$. The embeddings $H_{0}^{l} \subset H, H_{0}^{+l} \subset H^{+}$are dense; therefore we have the "rigged spaces" $H_{0}^{l} \subset H \subset\left(H_{0}^{l}\right)^{\prime}, H^{l} \subset H \subset\left(H^{l}\right)^{\prime}$, where $\left(H_{0}^{l}\right)^{\prime},\left(H^{l}\right)^{\prime}$ are the spaces of $H$ continuous linear functionals over $H_{0}^{l}$ and $H^{l}$, respectively (see, for example, 2]); there is a similar rigging for the "plus" spaces. The conjugate objects over the central topology will be called duals. Note that the notation for embeddings will be introduced below only as required. We also introduce the quotient spaces $H^{(l)}=H^{l} / H_{0}^{l}, H^{+(l)}=H^{+l} / H_{0}^{+l}$, which for convenience are represented as direct sums:

$$
H^{l}=H_{0}^{l} \oplus H^{(l)}, H^{+l}=H_{0}^{+l} \oplus H^{+(l)} .
$$

(We shall use the algebraic notation $\oplus$ for the direct sum, specifically stipulating orthogonality whenever it occurs.)

We also introduce the projection operators in a direct sum $p_{0}^{l}: H^{l} \rightarrow H_{0}^{l}, \gamma: H^{l} \rightarrow$ $H^{(l)}$ (quotient map) and the embedding operators $J_{0}^{l}: H_{0}^{l} \rightarrow H^{l}, J: H^{(l)} \rightarrow H^{l}$; there are also the analogous operators for the "plus" spaces: $p_{0}^{+l}: H^{+l} \rightarrow H_{0}^{+l}, \gamma^{+}: H^{+l} \rightarrow H^{+(l)}$ (quotient map) and the analogous embeddings $J_{0}^{+l}: H_{0}^{+l} \rightarrow H^{+l}, J^{+}: H^{+(l)} \rightarrow H^{+l}$. (The operators $J, J^{+}$have the sense of extension operators and can be constructed, for example, as the operators for determining the solutions of the Dirichlet problem for the corresponding vector system of polyharmonic equations of order $2 l$, if we regard the spaces $H^{(l)}$ and $H^{+(l)}$ as being in graded form, as in subsection $\mathrm{II}_{1}$ below.) In some cases we shall assume (and specifically mention) that

$$
\begin{array}{r}
N=N^{+}: H^{+}=H, \quad H^{+l}=H^{l}, \quad H_{0}^{+l}=H_{0}^{l}, \\
p_{0}^{+l}=p_{0}^{l}, \quad \gamma^{+}=\gamma, \quad J_{0}^{+l}=J_{0}^{l}, \quad J^{+}=J .
\end{array}
$$

Note that a reader only interested in the case of a scalar operator or an operator with square matrices as coefficients can forget about the pluses in the indices, apart from the indices attached to the letters $L, \mathcal{L}$ and $\Gamma$ (see $\$ 1.1$ ). One can also forget about these pluses if the original differential operation is formally selfadjoint. 
We shall also assume that:

$\left.\mathrm{II}_{1}\right)$. The spaces $H^{(l)}, H^{+(l)}$ are graded in the sense that we have the direct-sum decompositions

$$
\begin{aligned}
H^{(l)} & =H_{\partial}^{l-1 / 2} \oplus H_{\partial}^{l-3 / 2} \oplus \cdots \oplus H_{\partial}^{1 / 2}, \\
H^{+(l)} & =H_{\partial}^{+l-1 / 2} \oplus H_{\partial}^{+l-3 / 2} \oplus \cdots \oplus H_{\partial}^{+1 / 2},
\end{aligned}
$$

where

$$
\begin{aligned}
H_{\partial}^{k-1 / 2} & :=\left[H^{k-1 / 2}(\partial \Omega)\right]^{N} \subset H_{\partial}^{0}:=L_{2}^{N^{+}}(\partial \Omega), \\
H_{\partial}^{+k^{k-1 / 2}} & :=\left[H^{k-1 / 2}(\partial \Omega)\right]^{N^{+}} \subset H_{\partial}^{+0}:=L_{2}^{N^{+}}(\partial \Omega),
\end{aligned}
$$

$k=0,1, \ldots, l$, are Sobolev spaces of functions on $\partial \Omega$.

In view of the properties of the direct sum, there exist projection operators

$$
p_{j}: H^{(l)} \rightarrow H_{\partial}^{j+1 / 2}, \quad \gamma_{j}=p_{l-1-j} \circ \gamma: H^{l} \rightarrow H_{\partial}^{l-j-1 / 2}, \quad j=0,1, \ldots, l-1,
$$

and embedding operators

$$
J_{k}: H_{\partial}^{k+1 / 2} \rightarrow H^{l}, \quad k=0, \ldots, l-1,
$$

with the properties

$\gamma_{l-1-k} J_{k}=p_{k} \gamma J_{k}=\mathrm{id}_{H_{\partial}^{k+1 / 2}}, \quad \operatorname{id}_{H^{l}}=J_{0}^{l} p_{0}^{l}+\sum_{k=0}^{l-1} J_{k} p_{k} \gamma=J_{0}^{l}\left(\mathrm{id}_{H^{l}}-\gamma\right)+\sum_{k=0}^{l-1} J_{k} \gamma_{l-1-k}$

and analogous operators $p_{j}^{+}, \gamma_{j}^{+}, J_{k}^{+}$in the "plus" spaces. Note that the operators $J_{k}$ can be constructed as extension operators in the following way, for example: for $u \in H_{\partial}^{k+1 / 2}=$ $\left[H^{k+1 / 2}(\partial \Omega)\right]^{N}$ the equality $w=J_{k} u$ means that $w$ is a solution of the Dirichlet problem for the system of $N$ polyharmonic equations

$$
\Delta^{l} w=0,\left.\quad w\right|_{\partial \Omega}=0, \quad \forall j \neq l-k-1,\left.\quad \frac{\partial^{j} w}{\partial \nu^{j}}\right|_{\partial \Omega}=0,\left.\quad \frac{\partial^{l-k-1} w}{\partial \nu^{l-k-1}}\right|_{\partial \Omega}=u
$$

with normal $\nu$. Similarly, $w=J_{k}^{+} v$ means that $w$ is a solution of the same Dirichlet problem for the system of $N^{+}$polyharmonic equations. Note that we shall assume that the topology of $H_{\partial}^{0}$ is not related to the topology of the spaces $H$ and $H^{+}$. We shall denote the $H_{\partial}^{0}$-dual $\left(H_{\partial}^{+0}{ }^{0}\right.$-dual $)$ space to $H_{\partial}^{k+1 / 2}\left(H_{\partial}^{+k+1 / 2}\right)$ by $H_{\partial}^{-k-1 / 2}\left(H_{\partial}^{+-k-1 / 2}\right)$. Clearly, if conditions (0.1) hold, then

$$
H_{\partial}^{+i}=H_{\partial}^{i}, \quad p_{j}^{+}=p_{j}, \quad \gamma_{j}^{+}=\gamma_{j}, \quad J_{k}^{+}=J_{k} .
$$

$\mathrm{II}_{2}$ ). There exist continuous linear operators

$$
L_{(k)}: H^{l} \rightarrow H_{\partial}^{+0}, \quad L_{(k)}^{+}: H^{+l} \rightarrow H_{\partial}^{0}, \quad k=0, \ldots, l-1,
$$

associated with the operations $\mathcal{L}, \mathcal{L}^{+}$and with relations between them, which we call Green's formula: for any $u \in H^{l}, v \in H^{+l}$,

$$
(\mathcal{L} u, v)_{H^{+}}-\left(u, \mathcal{L}^{+} v\right)_{H}=\sum_{j=0}^{l-1}\left(L_{(l-1-j)} u, \gamma_{j}^{+} v\right)_{H_{\partial}^{+0}}=-\sum_{j=0}^{l-1}\left(\gamma_{j} u, L_{(l-1-j)}^{+} v\right)_{H_{\partial}^{0}} .
$$

Green's formula $(0.3)$ for the case $N=N^{+}$was obtained in the papers [38, 39. Namely, for smooth $u$ and $v$,

$$
\int_{\Omega}\left(L u \cdot \bar{v}-u \cdot \overline{L^{+} v}\right) d x=\sum_{q=0}^{m-1} \int_{\partial \Omega} L_{(m-q-1)} u \partial_{\nu}^{q} \bar{v} d s,
$$


where $L_{(p)}=\sum_{s=0}^{p} L_{(p s)} \partial_{\nu}^{s}$ is an operator of degree $p$ and $L_{(p s)}$ is a linear differential operator of degree $p-s$ along directions $\tau$ tangent to $\partial \Omega$ with smooth coefficients. Green's formula (0.4) for the case $N \neq N^{+}$is obtained via the same route and in the same form.

Thus, in (0.3),

$$
L_{(p)} u=\sum_{s=0}^{p} L_{(p s)} u \quad\left(L_{(p)}^{+} v=\sum_{s=0}^{p} L_{(p s)}^{+} v\right)
$$

are differential expressions of degree $k$, and $L_{(p s)}\left(L_{(p s)}^{+}\right)$are differential operators of order $p-s$ along directions $\tau$ tangent to $\partial \Omega$ with smooth $N^{+} \times N\left(N \times N^{+}\right)$-matrix coefficients dependent on the derivatives of the direction cosines of the outward normal $\nu(x)$ and on the derivatives of the coefficients $a_{\alpha}(x)\left(a_{\alpha}^{*}(x)\right)$ of the operation $\mathcal{L}\left(\mathcal{L}^{+}\right)$; $\gamma u=\gamma^{+} u=\left(\gamma_{0} u, \gamma_{1} u, \ldots, \gamma_{n-1} u\right), \gamma_{k} u=\gamma_{k}^{+} u=\left.\frac{\partial^{k} u}{\partial \nu^{k}}\right|_{\partial \Omega}$ with normal $\nu$. For brevity we denote the left-hand (solid) side of $(0.3)$ by $\left\langle L_{\partial} u, v\right\rangle_{H^{+}}=-\left\langle u, L_{\partial}^{+} v\right\rangle_{H}$, and we denote the right-hand (boundary) sides by $\left\langle\mathcal{L}_{\partial} \gamma u, \gamma^{+} v\right\rangle_{H_{\partial}^{+}}=-\left\langle\gamma u, \mathcal{L}_{\partial}^{+} \gamma^{+} v\right\rangle_{H_{\partial}}$, where

$$
H_{\partial}=\left[H_{\partial}^{0}\right]^{l}, \quad H_{\partial}^{+}=\left[H_{\partial}^{+0}\right]^{l}, \quad \mathcal{L}_{\partial}: H^{(l)} \rightarrow H_{\partial}^{+}, \quad \mathcal{L}_{\partial}^{+}: H^{+(l)} \rightarrow H_{\partial} .
$$

Remark 0.1. Note also that the operators $L_{(p p)}$ are of zero order:

$$
L_{(p p)} \gamma_{p} u=\left.(-1)^{l-1-p} \sum_{|\alpha|=l} a_{\alpha}(x)(-i \nu(x))^{\alpha} \frac{\partial^{p} u}{\partial \nu^{p}}\right|_{\partial \Omega},
$$

while the operator $\mathcal{L}_{\partial}$ is triangular with respect to the indices $p, s$; therefore for the case $N=N^{+}$, that is, under the conditions $(0.1),(0.2)$, the property that the kernel of the operator $\mathcal{L}_{\partial}$ be trivial is clearly satisfied, provided that

$$
\operatorname{meas}_{\partial \Omega}\left\{x \in \partial \Omega \mid \operatorname{det} \lambda_{l}(x,-i \nu(x))=0\right\}=0,
$$

where $\lambda_{l}(x, \xi)=\sum_{|\alpha|=l} a_{\alpha}(x) \xi^{\alpha}$ is the leading symbol (see Proposition 3.2 and Remark 3.1 regarding the fulfillment of $(0.5))$.

Remark 0.2. Note that we immediately obtain from Green's formula (0.3) the equality $(\mathcal{L} u, v)_{H^{+}}=\left(u, \mathcal{L}^{+} v\right)_{H}$ for $u \in H_{0}^{l}, v \in H^{+l}$. In fact, in this case $\gamma u=0$ and $\gamma_{j} u=0$, which hold when $u \in H^{l}, v \in H_{0}^{+l}$.

\section{Soluble extensions AND BOUndary-VAlue PRoblems}

1.1. The main definitions and conditions in the general theory of boundaryvalue problems. Here we recall the basic facts about extensions of a differential operator and boundary-value problems for differential equations in a domain (see [2, 18, 23, 45]).

We introduce the graph norm $\|u\|_{L}^{2}=\|u\|_{H}^{2}+\|\mathcal{L} u\|_{H^{+}}^{2}$, which is finite for elements of $H^{l}$. We denote the completions of the spaces $H^{l}, H_{0}^{l}$ in this norm by $D(\tilde{L}), D\left(L_{0}\right)$, respectively. They are Hilbert spaces and are densely embedded in $H$. Furthermore, the operator $\mathcal{L}$ admits an extension by continuity to the space $D(\tilde{L})$ in view of the estimate $\|\mathcal{L} u\|_{H^{+}} \leq\|u\|_{L}$. The restriction of this extension $\tilde{L}: D(\tilde{L}) \rightarrow H^{+}$to $D\left(L_{0}\right)$ is called the minimal extension (defined on $H_{0}^{l}$ ) of the operator $\mathcal{L}$ or simply the minimal operator and is denoted by $L_{0}$. Similarly we introduce the graph norm $\|v\|_{L^{+}}=\|v\|_{H^{+}}+\left\|\mathcal{L}^{+} v\right\|_{H}$, the spaces $D\left(\tilde{L}^{+}\right), D\left(L_{0}^{+}\right)$, the operator $\tilde{L}^{+}$and the minimal operator $L_{0}^{+}$for $\mathcal{L}^{+}$.

We also introduce the maximal extension of the operator $\mathcal{L}$ or simply maximal operator $L$ by the formula $L=\left(L_{0}^{+}\right)^{*}$, where the conjugate $*$ is taken in the sense of the theory of Hilbert spaces. Note that the operator $\tilde{L}$ is sometimes called the (other or second) maximal operator ([2, 23]). It is clear from the definition that $L$ is an extension of the operators $L_{0}$ and $\tilde{L}$; that is, its domain of definition $D(L)$ includes the spaces 
$D(\tilde{L})$ and $D\left(L_{0}\right)$ as closed subspaces and is a Hilbert space with norm $\|\cdot\|_{L}$. We define the maximal operator $L^{+}=L_{0}^{*}$ in a similar fashion. We introduce the boundary space as the quotient space $D(L) / D\left(L_{0}\right)$, and also the quotient map $\Gamma: D(L) \rightarrow C(L)$. The space $C(L)$ is a Hilbert space as $D\left(L_{0}\right)$ is a closed subspace in $D(L)$.

Consider the following conditions:

$$
\begin{gathered}
\text { the operator } L_{0}: D\left(L_{0}\right) \rightarrow H^{+} \text {has a continuous left inverse; } \\
\text { the operator } L_{0}^{+}: D\left(L_{0}^{+}\right) \rightarrow H \text { has a continuous left inverse; } \\
\qquad \begin{array}{c}
\tilde{L}=\left(L_{0}^{+}\right)^{*} \\
\tilde{L}^{+}=\left(L_{0}\right)^{*}
\end{array}
\end{gathered}
$$

Recall 26] that the existence of a continuous left inverse of a densely defined closed operator $T: H_{1} \supset D(T) \rightarrow H_{2}$ acting between Hilbert spaces is equivalent to the existence of an a priori estimate $\|u\|_{H_{1}} \leq C\|T u\|_{H_{2}}$.

In this case we say that the operator $T$ is correctly soluble. Thus, conditions (1.1), (1.2) imply the correct solubility of the corresponding operator; that is, the corresponding a priori estimate holds. Conditions (1.3) and (1.4) entail the corresponding equalities $D(\tilde{L})=D(L), D\left(\tilde{L}^{+}\right)=D\left(L^{+}\right)$, that is, the possibility of approximating each element in $D(L)$ or $D\left(L^{+}\right)$by elements of the smooth space $H^{l}$ or $H^{+l}$ in the corresponding graph norm. Conditions (1.1)-(1.4) were introduced in connection with the study of the notion of a well-posed boundary-value problem for a differential equation [2, 18, 23, 45, which we have already discussed. The actual concept of a general boundary-value problem differs among different authors. We consider the approaches of Vishik, Hörmander, Berezanskiu and Dezin, at the same time introducing the requisite definitions below. We merely note that in the papers $2,23,45$, they deal only with a scalar differential operator.

Following Vishik [21, it will be understood that we are given a boundary condition by indicating the domain of definition $D\left(L_{B}\right)$ of some operator $L_{B}$ that is the extension of the minimal operator $L_{0}$ and the restriction of the maximal operator

$$
L: D\left(L_{0}\right) \subset D\left(L_{B}\right) \subset D(L) .
$$

It is customary to call such operators extensions (of the operator $L_{0}$ ), and the extension $L_{B}: D\left(L_{B}\right) \rightarrow H^{+}$is said to be extendable if there exists a continuous two-sided inverse operator $L_{B}^{-}: H^{+} \rightarrow D\left(L_{B}\right), L_{B} L_{B}^{-1}=\mathrm{id}_{H}^{+}, L_{B}^{-1} L_{B}=\mathrm{id}_{D\left(L_{B}\right)}$. Clearly, such an operator solves the boundary-value problem $u \in D\left(L_{B}\right)$ for the equation $L u=f$ with any right-hand side $f \in H^{+}$. The operator $L_{1}: D\left(L_{1}\right) \rightarrow H^{+}$, which is the restriction of $L$, is called a soluble restriction if it has a continuous two-sided inverse. This inverse operator is a continuous right inverse of the operator $L$ and, conversely, each continuous right inverse $M$ of the operator $L$ induces a soluble restriction with domain of definition $D\left(L_{1}\right)=\operatorname{Im} M$, which is a soluble extension (of the operator $L_{0}$ ) if $D\left(L_{0}\right) \subset \operatorname{Im} M$. We also say that the extension $L_{B}:\left(L_{B}\right) \rightarrow H^{+}$is normally soluble if the image $\operatorname{Im} L_{B}$ is closed.

Following Hörmander [43, by a homogeneous boundary-value problem we mean the relations

$$
L u=f, \quad \Gamma u \in B,
$$

where $B \subset C(L)$ is the linear subspace of the boundary space defining the boundaryvalue problem. It is easy to see that a boundary condition of type $u \in D\left(L_{B}\right)$ gives rise to a condition $\Gamma u \in B$, where $B=D\left(L_{B}\right) / D\left(L_{0}\right)$, and, conversely, the space $B$ gives rise to an operator $L_{B}$ with domain of definition $D\left(L_{B}\right)=\Gamma^{-1}(B)$, which is the restriction of $L$ to the space $D\left(L_{B}\right)$ and the extension of the operator $L_{0}$, and which is closed if and only if $B$ is closed in $C(L)$ or $D\left(L_{B}\right)$ is closed in $D(L)$. The boundary-value problem 
(1.5) is said to be well-posed (we shall say correct), if the operator $L_{B}$ induced by it is extendable by an extension of $L_{0}$, that is, if the operator $L_{B}: D\left(L_{B}\right) \rightarrow H^{+}$has a continuous two-sided inverse.

In Berezanskiil's book 2, he considers boundary-value problems defined on a subspace $H^{l}(\mathrm{rp}), H_{0}^{l} \subset H^{l}(\mathrm{rp}) \subset H^{l}$. This subspace generates the domain of definition $D\left(L_{B}\right)$ of some extension of $L_{0}$ and restriction of $\tilde{L}$ obtained as the closure of the space $H^{l}(\mathrm{rp})$ in the graph norm $\|u\|_{L}$. We say that such a problem is smoothly generated. The definition of correctness is the same as above.

Dezin 23 regards it as natural to consider boundary-value problems generating extensions of the operator $L_{0}$ that are restrictions of $\tilde{L}$. Following him we shall call such extensions regular. Each smoothly generated problem gives rise to a regular extension, but the converse is false in general.

We now state the main results in the general theory of boundary-value problems.

Proposition 1.1 (Vishik [18] in Hörmander's interpretation [45]). The operator $L_{0}$ has a soluble extension and the operator $L$ has a correct boundary-value problem if and only if conditions (1.1) and (1.2) hold.

We note that the same conditions (1.1), (1.2) are equivalent to the existence of a correct boundary-value problem for the operator $L^{+}$. It also immediately follows from Proposition 1.1 that under the hypotheses $(1.1),(1.2),(1.3)$, the operator $L_{0}$ has a regular extension.

Proposition 1.2 (Vishik [18]). Under the conditions (1.1), (1.2),

1) in the space $D(L)$ there exists a closed subspace $W$ such that

$$
D(L)=D\left(L_{0}\right) \oplus \operatorname{ker} L \oplus W,
$$

where $\left.L\right|_{W}: W \rightarrow \operatorname{ker} L^{+}$is an isomorphism (one can write $W$ in the form $\left.L^{-1}\left(\operatorname{ker} L^{+}\right) \ominus \operatorname{ker} L\right)$.

2) In order that the extension $L_{B}$ be soluble it is necessary and sufficient that there exist a continuous operator $V: \operatorname{ker} L^{+} \rightarrow \operatorname{ker} L$ such that $D\left(L_{B}\right)=D\left(L_{0}\right) \oplus G$, where $G=\left\{w+\left.V L\right|_{W} w \mid w \in W\right\}$ is the graph of the operator $\left.V L\right|_{W}$.

Proposition 1.3 (Hörmander [43]). Under the hypotheses (1.1), (1.2), the boundaryvalue problem (1.5) is correct if and only if $C(L)=C(\operatorname{ker} L) \oplus B$, where $C(\operatorname{ker} L)=$ $\Gamma \operatorname{ker} L$.

We also consider the following conditions:

$$
\begin{aligned}
& \text { the operator } L: D(L) \rightarrow H^{+} \text {is surjective; } \\
& \text { the operator } L^{+}: D\left(L^{+}\right) \rightarrow H \text { is surjective; } \\
& \text { the operator } L_{0} \text { is normally soluble; } \\
& \text { the operator } L_{0}^{+} \text {is normally soluble. }
\end{aligned}
$$

It is clear that the surjectivity of a continuous operator $T: H_{1} \rightarrow H_{2}$ between Hilbert spaces is equivalent to the existence of a right continuous inverse for it. In this case the operator $T$ is said to be everywhere soluble.

To establish a connection between the conditions (1.6), (1.7) and the conditions (1.1), (1.2) we use the following well-known proposition (see [26]), stated for the case of different spaces of images and inverse images. 
Proposition 1.4. A closed linear operator $T: H_{1} \supset D(T) \rightarrow H_{2}$ between Hilbert spaces with dense domain $D(T)$

1) is correctly soluble if and only if the adjoint operator $T^{*}: H_{2} \supset D\left(T^{*}\right) \rightarrow H_{1}$ is everywhere soluble;

2) is normally soluble if and only if the adjoint operator is normally soluble.

The above proposition immediately gives rise to the following result.

Proposition 1.5. Condition (1.6) is equivalent to condition (1.2) and is equivalent to the two conditions: $\operatorname{ker} L_{0}=0$ and (1.9); condition (1.7) is equivalent to condition (1.1) and is equivalent to the two conditions: $\operatorname{ker} L_{0}^{+}=0$ and (1.8). Condition (1.8) is equivalent to the normal solubility of the operator $L^{+}$; condition (1.9) is equivalent to the normal solubility of the operator $L$.

The next result is an easy consequence of Propositions 1.1 and 1.2.

Proposition 1.6. Under conditions (1.1) and (1.2), each soluble extension of $L_{B}$ decomposes into a direct sum $L_{B}=L_{0} \oplus L_{B}^{\partial}$, where $L_{B}^{\partial}: B \rightarrow \operatorname{ker} L^{+}$is an isomorphism.

We now introduce some notation, which goes back to von Neumann, for the action of the maximal operators $L$ and $L^{+}$. We denote by $A$ the orthogonal complement of $D\left(L_{0}\right)$ in the Hilbert space $D(L)$ and we denote by $A^{+}$the orthogonal complement of $D\left(L_{0}^{+}\right)$ in $D\left(L^{+}\right)$.

Proposition 1.7 (31]).

1) $\left.L\right|_{A} A \subset A^{+} \quad \forall u \in A, L^{+} L u=-u ;\left.L\right|_{A}: A \rightarrow A^{+}$is a unitary isomorphism, $\left.L^{+}\right|_{A^{+}} A^{+} \subset A \quad \forall v \in A^{+}, L L^{+} v=-v ;\left.L^{+}\right|_{A^{+}}: A^{+} \rightarrow A$ is a unitary isomorphism.

2) There exists a unique isomorphism $\mathcal{L}_{C}: C(L) \rightarrow C\left(L^{+}\right)$such that

$$
(L u, v)_{H^{+}}-\left(u, L^{+} v\right)_{H}=\left(\mathcal{L}_{C} \Gamma u, \Gamma^{+} v\right)_{C\left(L^{+}\right)} .
$$

The proof is almost obvious if we observe that for $\mathcal{L}^{+}: H^{+} \rightarrow\left(\mathcal{D}^{\prime}(\Omega)\right)^{N}$ we have $\forall \phi \in(\mathcal{D}(\Omega))^{N}, \forall u \in A,\left\langle\mathcal{L}^{+} L u, \phi\right\rangle=(L u, L \phi)_{H^{+}}=-(u, \phi)_{H}$.

1.2. Examples of differential operators with the basic properties. We start with examples of scalar differential operators for which there exists a correct boundary-value problem. But first we give an example of an operator for which such a problem does not exist.

Since $\left.\operatorname{ker} \mathcal{L}\right|_{C_{0}^{\infty}(\Omega)} \subset \operatorname{ker} L_{0}$, the equalities

$$
\left.\operatorname{ker} \mathcal{L}\right|_{C_{0}^{\infty}(\Omega)}=\left.\operatorname{ker} \mathcal{L}^{+}\right|_{C_{0}^{\infty}(\Omega)}=0
$$

are necessary for the fulfillment of the conditions (1.1), (1.2).

The assertion of Theorem 13.6.15 in [43, due to Pliss, gives an example of an elliptic operator for which condition (1.10) fails. Thus, we have the following result.

Proposition 1.8. There exists an elliptic operator $\mathcal{L}$ of fourth order with $C^{\infty}$-coefficients $\mathbb{R}^{3}$, such that in some ball there does not exist a correct boundary-value problem.

It is well known that for a uniformly elliptic equation with homogeneous real symbol the Dirichlet problem is correct (see, for example, [30]). The validity of conditions (1.1), (1.2) for an operator with constant complex coefficients in any bounded domain was proved by Hörmander in [45]. Other proofs of this fact can be found in the papers [2, 23, 33.

We now give two further examples of classes of operators for which there is a correct boundary-value problem. In both cases the correctness follows from results in [52; see 
also 43. We recall that a differential operator $\mathcal{L}=\mathcal{L}(x, D)$ has constant strength 43 . in the domain $\bar{\Omega}$ if

$$
\forall x \in \bar{\Omega}, \quad \forall y \in \bar{\Omega}, \quad \exists C>0, \quad \forall \xi \in \mathbb{R}^{n}, \quad \frac{\tilde{P}(x, \xi)}{\tilde{P}(y, \xi)} \leq C,
$$

where

$$
|\tilde{P}(x, \xi)|^{2}=\sum_{|\alpha| \leq l}\left|D_{\xi}^{\alpha} \mathcal{L}(x, \xi)\right|^{2},
$$

and the operator with constant coefficients $P_{1}(D)$ is weaker than the same operator $P_{2}(D): P_{1} \prec P_{2}$ if $\tilde{P}_{1}(\xi) / \tilde{P}_{2}(\xi) \leq C$. An operator with constant strength can be represented in the form [44:

$$
\mathcal{L}(x, D)=P_{0}(D)+\sum_{j=1}^{N} C_{j}(x) P_{j}(D),
$$

where $C_{j} \in C^{\infty}(\bar{\Omega})$; the $P_{j}$ are operators forming a basis in a finite-dimensional vector space with constant coefficients that are weaker than $P_{0}: P_{j} \prec P_{0}$. In particular, formula (1.11) means that $D\left(L_{0}\right)=D\left(P_{0}\right)$. It follows from results of [52] (see also [43], Theorem 13.5.2) that for operators of constant strength conditions (1.6), (1.7) hold in some neighbourhood of each point. That conditions (1.6), (1.7) hold everywhere in the domain for operators of constant strength has been proved in the case when the coefficients are analytic in some wider domain (see 43, subsection 13.5). The same holds for operators of real principal type of the form (1.11), that is, if $P_{0}(\xi) \in \mathbb{R}[\xi]$, $\nabla P_{0} \neq 0$ for $\xi \neq 0$, ord $\mathrm{P}_{\mathrm{j}}<$ ord $\mathrm{P}_{0}$ (locally [45, in a domain [43] and [52, subsection $13.5])$.

The next two results are consequences of Propositions (1.1), (1.5).

Proposition 1.9. An operator of constant strength has a correct boundary-value problem in some neighbourhood of each point. If its coefficients are analytic in the domain $\tilde{\Omega}$, then it has a correct boundary-value problem in any domain $\Omega: \bar{\Omega} \subset \tilde{\Omega}$.

Proposition 1.10. An operator of real principal type of the form (1.11), where the $P_{j}$ are arbitrary operators of degree less than $m=\operatorname{deg} P=\operatorname{deg} \mathcal{L}$, has a correct boundary-value problem in a bounded domain.

For the case of matrix-valued differential equations in a bounded domain there are results on the existence of a correct boundary-value problem, firstly for uniformly elliptic (in the sense of Douglis-Nirenberg) quadratic systems (see, for example, 49]) in a domain with a smooth boundary, for which correct boundary-value problems have been studied and, secondly, quadratic systems of differential equations with constant coefficients satisfying the Paneah-Fuglede property: each minor $q$ of order $N-1$ of the characteristic matrix $p(\xi)$ satisfies the inequality

$$
|q(\xi)|^{2} \leq C \sum_{\alpha}\left|D^{\alpha} \operatorname{det} p(\xi)\right|^{2},
$$

which is necessary and sufficient for the fulfillment of conditions (1.1), (1.2) (see [36, 56]).

As regards conditions (1.3), (1.4), they hold:

- for each scalar uniformly elliptic operator in any domain with the cone condition (see [32]),

- for a hypoelliptic operator with constant coefficients in any domain 18, 
- for any scalar differential operator with constant coefficients in a domain with Hörmander's property $T: \Omega$ is a bounded domain and there exists a finite cover of the compact set $\bar{\Omega}$ by open sets $O_{i}$ such that $\forall i, \forall \epsilon>0, \exists t \in \mathbb{R}^{n}:|t|<\epsilon$, $\bar{\Omega} \cap O_{i}+t \subset \Omega[55$,

- for any scalar differential operator with constant coefficients, possibly with the addition of a component in the form of any differential operator with smooth coefficients of first order in a "normal" domain in the sense of Dezin [23,

- for any scalar differential operator of real principal type in any domain that is compactly embedded in the original domain $\Omega$ [23, 45.

In the case of matrix-valued operators in bounded domains, conditions (1.3), (1.4) have been systematically studied only for operators of type $\mathcal{L}=\nabla, \mathcal{L}=\left(\nabla, \nabla^{2}, \ldots, \nabla^{m}\right)$, $\mathcal{L}=\nabla^{\otimes m}$. In particular, for such operators these conditions hold in any domain that is star-shaped with respect to a point and, more generally, in a domain of class $C$ 32.

We also note the obvious fact that if condition (1.3) holds in the domain $\Omega$, then it holds in any subdomain $\Omega^{\prime} \subset \Omega$. We also note that if condition (1.6) holds in $\Omega$, then it also holds in any subdomain $\Omega^{\prime} \subset \Omega$ 23]. To prove this it suffices to extend an arbitrary function $f \in L_{2}\left(\Omega^{\prime}\right)$ by zero to a function $\tilde{f} \in L_{2}(\Omega)$, use condition (1.6) to find a function $u \in D(L), L u=f$ and take its restriction $\left.u\right|_{\Omega^{\prime}}$. Applying Propositions 1.1 and 1.5 , we obtain that the existence of a correct boundary-value problem in $\Omega$ implies the existence of such a problem in $\Omega^{\prime}$.

We note further that in a domain with property $T$ an operator with constant coefficients has the property $(L M)_{0}=L_{0} M_{0}$, and also that an example has been constructed of an operator with constant coefficients in a domain without condition $T$ in which property (1.3) fails [55.

We also draw attention to the concept of an induced boundary-value problem. If one is given a boundary-value problem (1.5) in a domain $\Omega$, then by the boundary-value problem induced in the domain $\Omega^{\prime} \subset \Omega$ we mean the problem $L v=\tilde{g}, \Gamma v \in B$, where $\tilde{g} \in L_{2}(\Omega)$ is the extension of $g$ by zero to $\Omega$ followed by the restriction of $v$ to $\Omega^{\prime}$. It is not difficult to show that the induced boundary-value problem is correct if the original one is 23 .

The next subsection is devoted to an outline of the proof of the correctness of a boundary-value problem.

\subsection{Construction of non-classical examples of correct boundary-value prob-} lems.

Proposition 1.11. Under conditions (1.1) and (1.2), every linear space $B \subset C(L)$ such that

1) $\Gamma^{-1} B \cap \operatorname{ker} L=0$,

2) there exists a continuous linear operator $M: \operatorname{ker} L^{+} \rightarrow D(L)$ with the properties

a) $L M=\left.\mathrm{id}\right|_{\operatorname{ker} L^{+}}$,

b) $\operatorname{Im} M \subset \Gamma^{-1} B$,

generates a correct boundary-value problem (1.5).

Proof. Let $L_{C}=\Gamma_{\operatorname{Im}} L \Gamma^{-1}: C(L) \rightarrow \operatorname{ker} L^{+}$, where $\Gamma_{\operatorname{Im}}: H \rightarrow \operatorname{ker} L^{+}$is the orthogonal projection. First we observe that the sum $M+L_{0}^{-1}: H^{+} \rightarrow D(L)$ is a continuous right inverse of the operator $L$ and the operator $\Gamma M$ is a continuous right inverse of the operator $L_{C}$. It follows from the properties of the direct sum that we have the directsum decomposition $C(L)=C(\operatorname{ker} L) \oplus B_{1}$, where $B_{1}=\operatorname{Im} \Gamma M$. Clearly $B \supset B_{1}$ and $B \cap C(\operatorname{ker} L)=0$. But this implies the equality $B=B_{1}$, because if an element $b \in B$ is such that $b \notin B_{1}$, then after factoring $\Gamma_{1}: C(L) \rightarrow C$ (ker $\left.L\right)$ along $B_{1}$ we obtain an element $\Gamma_{1} b \in C(\operatorname{ker} L)$ belonging to $B$, which gives a contradiction. 
Example 1.1. Consider the wave operator

$$
L=\square=\frac{\partial^{2}}{\partial x_{1} \partial x_{2}}, \quad \Omega=K=\left\{x \in \mathbb{R}^{2}|| x \mid<1\right\}
$$

in the disc $K$.

Proposition 1.12. The following boundary-value problem is correct in $L_{2}(K)$ :

$$
\begin{gathered}
\square u=f \in L_{2}(K), \\
\left.u\right|_{\Gamma_{1}}=0,\left.\quad u_{\nu}^{\prime}\right|_{\Gamma_{2}}=0,
\end{gathered}
$$

where $\Gamma_{1}=\left\{\frac{\pi}{2} \leq \tau \leq 2 \pi\right\}, \Gamma_{2}=\left\{\pi \leq \tau \leq \frac{3 \pi}{2}\right\}$, and $\tau$ is an angle variable.

Proof. As noted in $\$ 1.2$, conditions (1.1), (1.2) hold for a scalar differential operation with constant coefficients. Therefore Proposition 1.11 can be applied and, in view of the direct-sum decomposition $L=L_{0} \oplus L_{C}$, it suffices to take the right-hand side of (1.12) in the form $f=f_{1}\left(x_{1}\right)+f_{2}\left(x_{2}\right)$, where $f_{i}\left(x_{i}\right) \in L_{2}(K)$ means that $f_{i}(t) \in L_{2, \sigma}((-1,1))$ with weight $\sigma=\sqrt{1-t^{2}}$ since, as is easy to see,

$$
\left\|f_{i}\left(x_{i}\right)\right\|_{L_{2}(K)}^{2}=2\left\|f_{i}(t)\right\|_{L_{2, \sigma}((-1,1))}^{2} .
$$

The proof now consists of two parts:

1) The verification of the equality: $\Gamma^{-1} B \cap \operatorname{ker} L=0$. In fact, if

$$
\begin{aligned}
u_{1} \cos \tau+u_{2} \sin \tau & =0, & \frac{\pi}{2}<\tau<2 \pi, \\
\cos \tau u_{1}^{\prime} \cos \tau+\sin \tau u_{2}^{\prime} \sin \tau & =0, & \pi<\tau<\frac{3 \pi}{2},
\end{aligned}
$$

for some $u_{1}(x)=u_{1}\left(x_{1}\right), u_{2}(x)=u_{2}\left(x_{2}\right)$ in $D\left(\square_{0}\right) \subset H^{1}(K)$ (since $\square$ is an operator of real principal type; see $\$ 1.2)$, it follows that, firstly, after differentiating the first equation with respect to $\tau$ and comparing with the second equation we obtain that $u_{1}\left(x_{1}\right)=C_{1}=$ const for $x_{1}<0, u_{2}\left(x_{2}\right)=C_{2}=$ const for $x_{2}<0$ and, secondly, after considering the first equation we obtain that $u_{1}\left(x_{1}\right)=-C_{2}$ for $x_{1}>0$ and $u_{2}\left(x_{2}\right)=-C_{1}$ for $x_{2}>0$. But $u_{1}\left(x_{1}\right)$, which lies in $H^{1}(K)$, has a trace at the point $x_{1}=0$, therefore it is continuous at this point, so that we finally obtain $u=u_{1}+u_{2} \equiv 0$.

2) The verification of the fact that the operator

$$
f(x)=f_{1}\left(x_{1}\right)+f_{2}\left(x_{2}\right) \rightarrow x_{2} F_{1}\left(x_{1}\right)+x_{1} F_{2}\left(x_{2}\right)+\phi_{1}\left(x_{1}\right)+\phi_{2}\left(x_{2}\right),
$$

where $F_{i}$ is the primitive of $f_{i}$ and $V f=\phi_{1}\left(x_{1}\right)+\phi_{2}\left(x_{2}\right)$ will be chosen presently, is the operator $M: \operatorname{ker} L^{+} \rightarrow D(L)$ with properties 2a), 2b) in Proposition 1.11.

Property 2a) and the fact that $x_{2} F_{1}\left(x_{1}\right)+x_{1} F_{2}\left(x_{2}\right) \in L_{2}(K)$ are obvious. It merely remains to choose functions $\phi_{1}, \phi_{2}$ such that $\Gamma M f \in B$, that is, such that (for $x_{1}=\cos \tau$, $\left.x_{2}=\sin \tau\right)$

$$
\begin{array}{r}
x_{2} F_{1}\left(x_{1}\right)+x_{1} F_{2}\left(x_{2}\right)+\phi_{1}\left(x_{1}\right)+\phi_{2}\left(x_{2}\right)=0, \quad \frac{\pi}{2}<\tau<2 \pi \quad\left(\text { on } \Gamma_{1}\right) ; \\
x_{1} x_{2}\left[f_{1}\left(x_{1}\right)+f_{2}\left(x_{2}\right)\right]+x_{2} F_{1}\left(x_{1}\right)+x_{1} F_{2}\left(x_{2}\right)+x_{1} \phi_{1}\left(x_{1}\right)+x_{2} \phi_{2}\left(x_{2}\right)=0, \\
\pi<\tau<\frac{3 \pi}{2} \quad\left(\text { on } \Gamma_{2}\right) .
\end{array}
$$

Differentiating the first identity with respect to $\tau$ and comparing with the second, we obtain that on $\Gamma_{2}$,

$$
-\phi_{1}^{\prime}\left(x_{1}\right)=x_{2} f_{1}\left(x_{1}\right)+F_{2}\left(x_{2}\right), \quad-\phi_{2}^{\prime}\left(x_{2}\right)=x_{1} f_{2}\left(x_{1}\right)+F_{1}\left(x_{1}\right),
$$


whence

$$
\begin{aligned}
& \phi_{1}\left(x_{1}\right)=\int_{-1}^{x_{1}} \sqrt{1-x_{1}^{2}}\left[f_{1}\left(x_{1}\right)+F_{2}\left(-\sqrt{1-x_{1}^{2}}\right)\right] d x_{1}, \\
& \phi_{2}\left(x_{2}\right)=\int_{-1}^{x_{2}} \sqrt{1-x_{2}^{2}}\left[f_{2}\left(x_{2}\right)+F_{1}\left(-\sqrt{1-x_{2}^{2}}\right)\right] d x_{2} .
\end{aligned}
$$

We see that the functions $\phi_{1}, \phi_{2}$ are absolutely continuous on $[-1,0]$ and therefore lie in $L_{2}([-1,0])$. Next, we find the function $\phi_{1}$ on $(0,1)$ in the first identity considered on the ray $(3 \pi / 2,2 \pi)$ :

$$
-\phi_{1}\left(x_{1}\right)=\phi_{2}\left(-\sqrt{1-x_{1}^{2}}\right)-\sqrt{1-x_{1}^{2}} F_{1}\left(x_{1}\right)+x_{1} F_{2}\left(-\sqrt{1-x_{1}^{2}}\right)
$$

and we find the function $\phi_{2}$ on $(0,1)$ in the same first identity considered on the ray $(\pi / 2, \pi)$ :

$$
-\phi_{2}\left(x_{2}\right)=\phi_{1}\left(-\sqrt{1-x_{2}^{2}}\right)-\sqrt{1-x_{2}^{2}} F_{2}\left(x_{2}\right)+x_{2} F_{1}\left(-\sqrt{1-x_{2}^{2}}\right) .
$$

We have determined the function $V f \in \operatorname{ker} L \subset D(L)$; this completes the proof.

We merely observe that the operator $V$ is the Vishik operator of this problem (see Proposition 1.2) with respect to the boundary-value problem induced in $K$ (see the end of $\S 1.2)$ by the Goursat problem: $\left.u\right|_{x_{1}=0}=0,\left.u\right|_{x_{2}=0}=0$.

\section{Boundary PROPERTIES OF SOLUTIONS}

Let $f \in H^{+}$. We consider the equation

$$
L u=f,
$$

where $L$ is the maximal operator generated by the operation $\mathcal{L}$. In the preceding section we used the propositions in subsection I of $\S 0$. In this section we adopt the assumptions of subsections I, II of $\S 0$. We want to clarify here which boundary properties each solution of equation (2.1) has; we mean by this which of the spaces associated with the gradation the trace expressions generated by Green's formula belong to. If we look at the case of a differential operator, then we will in fact be answering the question: do there exist any traces of the solutions in $L_{2}(\Omega)$ of equation (2.1) with right-hand side in $L_{2}(\Omega)$, if only in the sense of distributions (see Example 2.1 below) and what are their properties?

2.1. Associated traces of a solution. Properly speaking, the situation considered in the previous section has changed only in that, along with the gradation, we now have Green's formula (0.3) for elements $u \in H^{l}, v \in H^{+l}$, which we write here in the form

$$
G(u, v)=\left(L_{\partial} u, v\right)_{H^{+}}=\sum_{j=0}^{l-1}\left(L_{(l-j-1)} u, \gamma_{j}^{+} v\right)_{H_{\partial}^{+0}} .
$$

First we note that the Green's function $G(u, v)$ can be extended to the space $D(L) \otimes$ $D\left(L^{+}\right)$by the formula

$$
G(u, v)=(L u, v)_{H^{+}}-\left(u, L^{+} v\right)_{H}
$$

in view of the inequality

$$
|G(u, v)| \leq\|u\|_{D(L)}\|v\|_{D\left(L^{+}\right)},
$$

which implies, firstly, the continuity of the Green's functional extended in this way, and secondly, the continuity of the operators $L_{\partial}: D(L) \rightarrow D^{\prime}\left(L^{+}\right)$and $L_{\partial}^{+}: D\left(L^{+}\right) \rightarrow D^{\prime}(L)$, defined by the formula

$$
G(u, v)=\left(L_{\partial} u, v\right)_{H^{+}}=-\left(u, L_{\partial}^{+} v\right)_{H} .
$$


We now show that by using formula (2.2), the operators $L_{(l-j-1)}$ can be continuously extended to the domain of definition of the operator $D(\tilde{L})$, so that for each element in $D(\tilde{L})$ there exist trace expressions $L_{(l-j-1)} u$. Let $\psi \in H_{\partial}^{+l-k-1 / 2}$ and $u \in H^{l}$; we substitute $v=J_{l-k-1}^{+} \psi$ in formula (2.2) (for the definition of the operator $J_{k}^{+}$, see subsection $\mathrm{II}_{1}$ of the main scheme in $\S 0.1$ ), and we obtain

$$
\left(L_{\partial} u, J_{l-k-1}^{+} \psi\right)_{H^{+}}=\left(L_{(l-k-1)} u, \psi\right)_{H_{\partial}^{+0}},
$$

since $\gamma_{k}^{+} J_{l-k-1}^{+}=\mathrm{id}_{H^{+} l-k-1 / 2}$. Estimating the left-hand side of this equality with the help of (2.4) and taking into account the continuity of $J_{l-k-1}^{+}$and the embedding $H^{+l} \rightarrow$ $D\left(L^{+}\right)$, we obtain the inequality

$$
\left|\left(L_{(l-k-1)} u, \psi\right)_{H_{\partial}^{+0}}\right| \leq C\|u\|_{D(L)}\|\psi\|_{H_{\partial}^{+l-k-1 / 2}},
$$

which enables us to continuously extend the operator $L_{(l-k-1)}: H^{l} \rightarrow H_{\partial}^{+0}$ to a continuous operator $L_{(l-k-1)}: D(\tilde{L}) \rightarrow H_{\partial}^{+-l+k+1 / 2}$, where $H_{\partial}^{+-l+k+1 / 2}$ is the dual space of $H_{\partial}^{+l-k-1 / 2}$ in the sense of the topology of $H_{\partial}^{+0}$. Since the embedding $H^{l} \subset D(\tilde{L})$ is dense, this extension to $D(\tilde{L})$ is unique.

Definition 2.1. For each $u \in D(\tilde{L})$ and each $k=0,1, \ldots, l-1$ we obtain a continuous linear functional $L_{(k)} u \in H_{\partial}^{+-k-1 / 2}$, which we call the $k$-th trace of the element $u \in D(\tilde{L})$ associated with the operator $L$, or simply the $k$ th $L$-trace of the element $u$.

In view of inequality (2.6) the associated traces depend continuously on $u \in D(\tilde{L})$; therefore if $u \in D(\tilde{L})$ and the sequence $w_{j} \in H^{l}$ converges to $u$ in $D(L)$, the sequence $L_{(k)} w_{j}$ converges to $L_{(k)} u$ in the space $H_{\partial}^{+-k-1 / 2}$. Here it is clear that the limiting $L$-trace does not depend on the choice of the approximating sequence $w_{j}$.

We now summarize our construction in the form of the following proposition.

Proposition 2.1. Each element $u$ in the domain of definition $D(\tilde{L})$ of the operator $\tilde{L}$ has $L$-traces $L_{(k)} u \in H_{\partial}^{+-k-1 / 2}, k=0,1, \ldots, m-1$, such that for every sequence $w_{j} \in H^{l}$, converging to an element $u$ in $D(\tilde{L})$, the sequence $L_{(k)} w_{j} \in H_{\partial}^{+0}$ converges in the space $H_{\partial}^{+-k-1 / 2}$ to an element $L_{(k)} u$ that is continuously dependent on $u$ but is independent of the choice of the sequence $w_{j}$.

Next, since we want to have unique extensions of the functionals $L_{(k)}$ from the closed subspace $D(\tilde{L})$ to $D(L)$, we shall assume below that the extension is realized with preservation of the norm; that is, we shall define the functionals $L_{(k)}$ to be zero on the orthocomplement of $D(\tilde{L})$ in $D(L)$; similarly we shall define $L_{(k)}^{+}$to be zero on the orthocomplement of $D\left(\tilde{L}^{+}\right)$in $D\left(L^{+}\right)$. Thus the functional $L_{(k)}$ is defined on the space $D(L)$, while the functional $L_{(k)}^{+}$is defined on the space $D\left(L^{+}\right)$.

Example 2.1. For the case of a scalar differential operator $\mathcal{L}$ the existence of associated traces in the distributions for functions in $D(\tilde{L})$ was proved in 5, 11. If $\mathcal{L}=-\Delta$ is the Laplace operator, then $L_{(0)} u=\left.u\right|_{\partial \Omega}, L_{(1)} u=-\left.u_{\nu}^{\prime}\right|_{\partial \Omega}$. The fact that $\left.u\right|_{\partial \Omega} \in H^{-1 / 2}(\partial \Omega)$ for $u \in D(-\Delta)$ had, in fact, already been proved by Vishik [18. It is known 39] that if part of the boundary $\partial^{\prime} \Omega$ does not have characteristic points, that is, points of tangency of real characteristic surfaces (as, for example, in the elliptic case), then ordinary traces $\left.u\right|_{\partial^{\prime} \Omega},\left.u_{\nu}^{\prime}\right|_{\partial^{\prime} \Omega}, \ldots,\left.u_{\nu^{l}}^{(l)}\right|_{\partial^{\prime} \Omega}$ exist for each solution in $L_{2}(\Omega)$ of the differential equation (2.1). However, examples show that such traces for solutions in $L_{2}(\Omega)$ do not exist in $D^{\prime}(\partial \Omega)$ 
even for the simplest equations. For example, for the wave equation

$$
L u=\square u=\frac{\partial^{2} u}{\partial x_{1} \partial x_{2}}=0
$$

in the unit disc $K=\left\{x \in \mathbb{R}^{2} \mid x^{2}<1\right\}$ the solution $u(x)=\left(1-x_{1}^{2}\right)^{-5 / 8}$ belongs to $L_{2}(K)$, but $\left\langle\left. u\right|_{\partial K}, 1\right\rangle=\infty$ in the sense that

$$
\lim _{r \rightarrow 1-0} \int_{|x|=r} u(x) d s_{x}=\infty,
$$

so that the trace $\left.u\right|_{\partial K}$ is not a distribution. However, it can be shown [16] that for each solution $u \in L_{2}(K)$ of the original equation $L u=0$ the trace of the product

$$
L_{(0)} u:=-\left.\lambda(x) u(x)\right|_{\partial K} \in L_{2}(\partial K)
$$

exists, where $\left.\lambda(x)\right|_{\partial K}=\lambda(\nu)=x_{1} x_{2}$ is the symbol of the operator $L$. In fact, we take

$$
\chi=\left.x_{1} x_{2} u(x)\right|_{\partial K}=\sin \tau \cos \tau\left[u_{1}(\cos \tau)+u_{2}(\sin \tau)\right]=\chi_{1}+\chi_{2},
$$

where $\tau$ is an angle coordinate. Then

$$
\begin{aligned}
\left\langle\chi_{1}, \varphi\right\rangle_{\partial K} & =\left|\int_{0}^{\pi} \sin \tau \cos \tau u_{1}(\cos \tau)[\varphi(\tau)-\varphi(-\tau)] d \tau\right| \\
& \leq 2\|\varphi\|_{L_{\infty}(\partial K)} \int_{-1}^{1}\left|u_{1}\left(x_{1}\right)\right| d x_{1} \leq C\|\varphi\|_{L_{\infty}(\partial K)}\|u\|_{L_{2}(K)}<\infty
\end{aligned}
$$

and similarly for $\chi_{2}$.

In exactly the same way, not all solutions have a trace $\left.u_{\nu}^{\prime}\right|_{\partial K}$, but for each solution $u \in L_{2}(K)$ there exists the trace expression

$$
L_{(1)} u=\lambda(x) u_{\nu}^{\prime}(x)+\lambda_{\tau}^{\prime} u_{\tau}^{\prime}+\left.\frac{1}{2} \lambda_{\tau \tau}^{\prime \prime} u\right|_{\partial K} \in H^{-3 / 2}(\partial K) .
$$

We note 14] that the same traces $L_{(0)} u=-\lambda(\nu(x)) u(x)$ and $L_{(1)} u$, where

$$
\lambda(x)=a x_{1}^{2}+b x_{1} x_{2}+c x_{2}^{2},
$$

exist for each solution $u \in L_{2}(K)$ of the equation

$$
L u=a u_{x_{1} x_{1}}+b u_{x_{1} x_{2}}+c u_{x_{2} x_{2}}=0
$$

with constant complex, and even matrix-valued, coefficients for $u \in D(L)$ in the disc $K$. Proposition 2.1 shows that these $L$-traces, the expressions for which are obtained from the corresponding Green's formula, exist for each function $u \in D(\tilde{L})$.

2.2. Spaces of associated traces, and the boundary operator. Let $u \in D(\tilde{L})$ and suppose that the sequence $u_{k} \in H^{l}$ approximates $u$. Substituting $u_{k}$ for $u$ in (2.2), (2.3) and passing to the limit, in view of Proposition 2.1, we obtain the following result.

Proposition 2.2. For each pair $u \in D(\tilde{L}), v \in H^{+l}$, Green's formula holds with associated traces on the right-hand side

$$
(L u, v)_{H^{+}}-\left(u, L^{+} v\right)_{H}=\sum_{k=0}^{l-1}\left\langle L_{(l-k-1)} u, \gamma_{k}^{+} v\right\rangle_{H_{\partial}^{+0}} .
$$

The next result is an immediate consequence of the above formula and the definition of the adjoint operator in Hilbert spaces.

Proposition 2.3. An element $u \in D(\tilde{L})$ has trivial L-traces $L_{(k)} u=0, k=0,1, \ldots$, $l-1$, if and only if it belongs to $D\left(\tilde{L}^{+*}\right)$. 
It is clear that if (1.4) holds, that is, $D\left(\tilde{L}^{+}\right)=D\left(L^{+}\right)$, then $D\left(\tilde{L}^{+*}\right)=D\left(L_{0}\right)$; therefore we have the following result.

Proposition 2.4. Condition (1.4) is equivalent to the assertion that an element in $D(\tilde{L})$ belongs to the space $D\left(L_{0}\right)$ if and only if it has regular L-traces. Condition (1.3) is equivalent to the assertion that an element in $D\left(\tilde{L}^{+}\right)$belongs to the space $D\left(L_{0}^{+}\right)$if and only if it has regular $L^{+}$-traces.

Apart from that, if $u \in D\left(L_{0}\right) \subset D(\tilde{L})$ this entails the triviality of its $L$-traces, even without condition (1.4) in view of formula (2.7). Hence we have the following result.

Proposition 2.5. In order that an element $u \in D(L)$ belong to $D\left(L_{0}\right)$ it is necessary, and under conditions (1.3), (1.4) sufficient, that it have regular L-traces $L_{(k)} u=0$, $k=0,1, \ldots, l-1$.

The above assertion enables one to restrict the associated traces to the quotient space of $D(L)$, namely, the boundary space $C(L)=D(L) / D\left(L_{0}\right)$, since $\forall u_{0} \in D\left(L_{0}\right)$, $L_{(k)}\left(u+u_{0}\right)=L_{(k)} u$. In what follows we shall denote by $H^{(-l)}$ the $H_{\partial}$-conjugate space of $H^{(l)}$, that is, $H^{(-l)}=H_{\partial}^{-l+1 / 2} \oplus \cdots \oplus H_{\partial}^{-1 / 2}$; the analogous notation is $H^{+(-l)}$.

Definition 2.2. We denote by

$$
\mathcal{L}_{C, k}\left(u+D\left(L_{0}\right)\right)=\mathcal{L}_{C, k} \Gamma u=L_{(k)} u \in H_{\partial}^{+-k-1 / 2}, \quad k=0, \ldots, l-1,
$$

the restriction of $L_{(k)}$ to the quotient space $C(L)$. Then, in accordance with Propositions 2.1 and 2.5, the system of operators

$$
\mathcal{L}_{C}=\left\{\mathcal{L}_{C, k}: C(L) \rightarrow H_{\partial}^{+}{ }^{-k-1 / 2} \mid k=l-1, \ldots, 0\right\}=\left\{\mathcal{L}_{C, l-1}, \ldots, \mathcal{L}_{C, 0}\right\}
$$

is a continuous operator $\mathcal{L}_{C}: C(L) \rightarrow H^{+(-l)}$, and for each $\Gamma u \in C(L)$ the functional $\mathcal{L}_{C} \Gamma u \in H^{+}(-l)$ on the element $w=\left(w_{0}, \ldots, w_{l-1}\right)$ in $H^{+(l)}, w_{j} \in H_{\partial}^{+l-j-1 / 2}$, acts in accordance with the formula

$$
\left\langle\mathcal{L}_{C} \Gamma u, w\right\rangle_{H_{\partial}^{+}}=\sum_{k=0}^{m-1}\left\langle L_{(l-1-k)} u, w_{k}\right\rangle_{H_{\partial}^{+0}} .
$$

The operator $\mathcal{L}_{C}: C(L) \rightarrow H^{+}(-l)$ is called the boundary operator for the operator $\mathcal{L}$.

The boundary operator $\mathcal{L}_{C}^{+}: C\left(L^{+}\right) \rightarrow H^{(-l)}$ for the operator $\mathcal{L}^{+}$is defined similarly.

Note that for smooth traces, that is, on the space $H^{(l)}$, the operator $\mathcal{L}_{C}$ coincides with the operator $\mathcal{L}_{\partial}$ in subsection $\mathrm{II}_{2}$ of $\S 0$.

Definition 2.3. We call the image $\operatorname{Im} \mathcal{L}_{C} \subset H^{+(-l)}$ with the strongest topology in which the map $\mathcal{L}_{C}$ is continuous the space of associated traces of the operator $L$ or the space of $L$-traces, which we denote by $A(L)$; its elements are $\mathcal{L}_{C} u=\left\{L_{(k)} u\right\} \in H^{+(-l)}$. We also denote by $A(\operatorname{ker} L)$ the space of associated traces of elements of the kernel of $L$. The spaces $A\left(L^{+}\right)$and $A\left(\operatorname{ker} L^{+}\right)$are defined similarly.

On the other hand, the form $\left\langle\mathcal{L}_{C} \Gamma u, \Gamma^{+} v\right\rangle$, defined on the elements $\Gamma u \in C(\tilde{L})$, $v \in H^{+(l)}$, can be continuously extended to a form $\left\langle\hat{\mathcal{L}}_{C} \Gamma u, \Gamma^{+} v\right\rangle$ on the space $C(\tilde{L}) \otimes$ $C\left(\tilde{L}^{+}\right)$(but not on the space $C(L) \otimes C\left(\tilde{L}^{+}\right)$, since extensions of operators $L_{(k)}$ to $D(L)$ have already been chosen for the convenience of the exposition) in accordance with the formula

$$
\left\langle\hat{\mathcal{L}}_{C} \Gamma u, \Gamma^{+} v\right\rangle=\langle L u, v\rangle_{H^{+}}-\left\langle u, L^{+} v\right\rangle_{H}
$$

in view of the property

$$
\left\langle L_{\partial}\left(u+u_{0}\right), v+v_{0}\right\rangle_{H^{+}}=\left\langle L_{\partial} u, v\right\rangle_{H^{+}}, \quad \forall u_{0} \in D\left(L_{0}\right), \quad \forall v_{0} \in D\left(L_{0}^{+}\right)
$$


and the inequality

$$
\left\langle\hat{\mathcal{L}}_{C} \Gamma u, \Gamma^{+} v\right\rangle \leq\|\Gamma u\|_{C(L)}\left\|\Gamma^{+} v\right\|_{C\left(L^{+}\right)},
$$

which is a consequence of (2.4). Now the continuity of this form brings about the continuity of the functional of $\hat{\mathcal{L}}_{C} \Gamma u$ on the space $C\left(\tilde{L}^{+}\right)$and the continuity of the action of the operator $\hat{\mathcal{L}}_{C}: C(\tilde{L}) \rightarrow C^{*}\left(\tilde{L}^{+}\right)$.

Remark 2.1. Note that the exposition in $\S \S 2.1$ and 2.2 was conducted in such a way that going over to the Banach case presented no special difficulty. In this connection we also used the notation $C^{*}\left(\tilde{L}^{+}\right)$.

Remark 2.2. Note that so far we have no justification in assuming that the inclusion $C^{*}\left(\tilde{L}^{+}\right) \subset H^{+(-l)}$ holds, even if we suppose that the inclusion $H^{+(l)} \subset C\left(\tilde{L}^{+}\right)$holds, since the topology of $H_{\partial}^{+0}$, with respect to which the space $H^{+(-l)}$ is dual to the space $H^{+(l)}$, is in no way related to the topology of $H$ or the topology of $H^{+}$, as pointed out in subsection II of $\S 0$. Furthermore, the space $C^{*}\left(\tilde{L}^{+}\right)$is in general not related to the space $C^{\prime}\left(\tilde{L}^{+}\right)$, which is the $H^{+}$-conjugate of the space $C\left(\tilde{L}^{+}\right)$, embedded as a subspace of $D\left(L^{+}\right)$. Jumping ahead, we observe that in our view, the proper place for these spaces is indicated in the constructions of $\S 4$; the first step in this direction is Proposition 2.8 below.

Next, the space $H^{+(l)}$ under the action of the operator $\Gamma^{+} I^{+} J^{+l}$ with the embedding $I^{+}: H^{+l} \rightarrow D(L)$ is continuously mapped into the space $C\left(L^{+}\right)$; clearly the map $\Gamma^{+} I^{+} J^{+l}$ is an injection if and only if

$$
D\left(L_{0}^{+}\right) \cap I^{+} J^{+l} H^{+(l)}=0 \text {, which is equivalent to } \operatorname{ker} \mathcal{L}_{\partial}^{+}=0 .
$$

Furthermore, we have the identity

$$
\left\langle\hat{\mathcal{L}}_{C} \Gamma u, \Gamma^{+} I^{+} J^{+l} w\right\rangle=\left\langle\mathcal{L}_{C} \Gamma u, w\right\rangle_{H_{\partial}^{+}}, \quad \forall u \in D(L), \quad \forall w \in H^{+(l)},
$$

from which it follows that

$$
\left(\Gamma^{+} I^{+} J^{+l}\right)^{*} \hat{\mathcal{L}}_{C} \Gamma u=\mathcal{L}_{C} \Gamma u .
$$

Condition (1.4) ensures that the image of $I^{+}$is dense in $C\left(L^{+}\right)$as is the image of $\Gamma^{+} I^{+} J^{+l}$. Thus, in this case the operator $\left(\Gamma^{+} I^{+} J^{+l}\right)^{*}$ is not only the operator of substitution into a functional but also the operator of a dense embedding. Hence it is itself a dense embedding. We have thus established the following result.

Proposition 2.6. Under the conditions (1.4) and (2.11) the space $C^{*}\left(\tilde{L}^{+}\right)$can be embedded with its topology in $A(L)$ and the embeddings $C^{*}\left(\tilde{L}^{+}\right) \subset A(L) \subset H^{+(-l)}$ are dense.

Similarly $C^{*}(\tilde{L}) \subset A\left(L^{+}\right) \subset H^{(-l)}$ with dense embeddings under condition (1.3) and the condition

$$
D\left(L_{0}\right) \cap I J^{l} H^{(l)}=0 \text {, which is equivalent to the assertion that } \operatorname{ker} \mathcal{L}_{\partial}=0,
$$

where $I: H^{l} \subset D(L)$ is an embedding.

2.3. Some properties of boundary operators. We now look at the kernels of the operators $\mathcal{L}_{C}, \mathcal{L}_{C}^{+}$. Parts 1) and 2) of the next proposition are immediate consequences of Propositions 2.3, 2.4, 2.5 and the definition of the operators $\mathcal{L}_{C}, \mathcal{L}_{C}^{+}$.

\section{Proposition 2.7.}

1) The kernel of the restriction $\left.\mathcal{L}_{C}\right|_{C(\widetilde{L})}$ of a bounded operator $\mathcal{L}_{C}$ coincides with the quotient space

$$
\left(D\left(\tilde{L}^{+*}\right) \cap D(\tilde{L})\right) / D\left(L_{0}\right) .
$$

Under condition (1.4) this kernel is trivial. 
2) The kernel of the restriction $\left.\mathcal{L}_{C}^{+}\right|_{C\left(\widetilde{L}^{+}\right)}$coincides with the quotient space

$$
\left(D\left(\tilde{L}^{*}\right) \cap D\left(\tilde{L}^{+}\right)\right) / D\left(L_{0}^{+}\right) .
$$

Under condition (1.3) this kernel is trivial.

3) Suppose that condition (2.12) holds. Then the condition

$$
\text { the lineal } H^{l} \cap D\left(\tilde{L}^{+*}\right) \text { is dense in } D\left(\tilde{L}^{+*}\right)
$$

is equivalent to condition (1.4).

4) Suppose that condition (2.11) holds. Then the condition

$$
\text { the lineal } H^{+l} \cap D\left(\tilde{L}^{*}\right) \text { is dense in } D\left(\tilde{L}^{*}\right)
$$

is equivalent to condition (1.3).

The proofs of parts 3) and 4) are obtained from parts 1) and 2). In fact, for each smooth element $v \in H^{l} \cap D\left(\tilde{L}^{+*}\right)$ we have $\Gamma v \in \operatorname{ker} \mathcal{L}_{C}$ in view of part 1$)$, and $\Gamma v=\gamma v=0$ in view of (2.12); therefore $H^{l} \cap D\left(\tilde{L}^{+*}\right)=H_{0}^{l}$. But by condition (1.4) the smooth elements are dense in the space $D\left(\tilde{L}^{+*}\right)$; hence the latter coincides with $D\left(L_{0}\right)$, which is required for the proof of the necessity in part 3). The sufficiency is obvious. Part 4) is proved in the same way.

Note that conditions (2.11) and (2.12) hold under the assumption (0.5) in $\S 0$ (see $\S 3.1$ below).

Apart from that the question of whether the image spaces of the operators $\mathcal{L}$ and $\mathcal{L}^{+}$ are closed remains open. We shall return to the study of these images.

Suppose that hypotheses (1.3), (1.4) hold. Then the operator $\mathcal{L}_{C}$, defined by formula (2.8) and extended to the space $C(\tilde{L}) \otimes C\left(\tilde{L}^{+}\right)=C(L) \otimes C\left(L^{+}\right)$by formula $(2.9)$, satisfies the conditions of part 3 of Proposition 1.7. The next result follows from parts 3,4 of this proposition.

Proposition 2.8. Suppose that conditions (1.3) and (1.4) hold. Then

1) the spaces $A(L)$ and $A\left(L^{+}\right)$can be identified with the spaces $C^{*}\left(L^{+}\right)$and $C^{*}(L)$, respectively; therefore, $C^{*}\left(L^{+}\right) \subset H^{(-l)}, C^{*}(L) \subset H^{(-l)}$;

2) the operators $\mathcal{L}_{C}: C(L) \rightarrow C^{*}\left(L^{+}\right), \mathcal{L}_{C}^{+}: C\left(L^{+}\right) \rightarrow C^{*}(L)$ are isomorphisms;

3) after the Riesz identification $C^{*}\left(L^{+}\right)=C\left(L^{+}\right), C^{*}(L)=C(L), D^{*}\left(L^{+}\right)=$ $D\left(L^{+}\right), D^{*}(L)=D(L)$ we have the formulae

$$
\Gamma L^{+} \Gamma^{+*}=\mathcal{L}_{C}^{-1}, \quad \Gamma^{+} L \Gamma^{*}=\mathcal{L}_{C}^{+-1} .
$$

We now consider the $L$-traces of elements of the kernel of the maximal operator $L$.

Proposition 2.9. Suppose that conditions (1.3), (1.4) and (1.8) hold. Then

1) the element $\Gamma u \in C(L)$ lies in the space $C(\operatorname{ker} L)$ if and only if

$$
\forall \Gamma^{+} v \in C\left(\operatorname{ker} L^{+}\right), \quad\left\langle\mathcal{L}_{C} \Gamma u, \Gamma^{+} v\right\rangle_{C\left(L^{+}\right)}=0 ;
$$

2) if, in addition, the following condition holds:

$$
\text { the smooth kernel } \tilde{K}^{+}=\operatorname{ker} L^{+} \cap H^{l} \text { is dense in the kernel } \operatorname{ker} L^{+} \subset H^{+} \text {, }
$$

then the $L$-traces of an element $u \in D(L)$ belong to the space $A(\operatorname{ker} L)$ if and only if for every element $v \in \tilde{K}^{+}=\operatorname{ker} L^{+} \cap H^{l}$ the following condition holds:

$$
\sum_{j=0}^{l-1}\left\langle L_{(l-1-j)} u, \gamma_{j}^{+} v\right\rangle_{H_{\partial}^{+0}}=0 .
$$


Proof. 1) From equality (2.9) for the elements $\Gamma u \in C(\operatorname{ker} L)$ and $\Gamma^{+} v \in C\left(\operatorname{ker} L^{+}\right)$we obtain (2.15). Conversely, if we are given the identity (2.15), then it follows from (2.9) that $\forall v \in \operatorname{ker} L^{+},\langle L u, v\rangle_{H^{+}}=0$, that is, $L u \perp \operatorname{ker} L^{+}$, and from condition (1.10) we then obtain the existence of an element $u_{0} \in D\left(L_{0}\right)$ such that $L u=L_{0} u_{0}$. Therefore, the element $U=u-u_{0}$ belongs to the kernel ker $L$ and has the same $L$-traces as the element $u$, that is, $\Gamma U \in C(\operatorname{ker} L)$.

Part 2) follows from part 1) and equalities (2.7), (2.9). This completes the proof.

Note that condition (2.17) is the starting point for the study of the properties of boundary-value problems for specific equations [4, 6, 8, 13, 14, 15, 16,

We now establish a connection between the operators $\hat{\mathcal{L}}_{C}$ and $\hat{\mathcal{L}}_{C}^{+}$and the boundary components $L_{C}$ and $L_{C}^{+}$of the maximal operators $L$ and $L^{+}$.

Proposition 2.10. Suppose that conditions (1.10), (1.11) hold, the operators $\hat{\Gamma}, \hat{\Gamma}^{+}$, $\hat{\Gamma}_{\operatorname{Im}}$ and $\hat{\Gamma}_{\operatorname{Im}}^{+}$, which are respectively some right inverses of $\Gamma, \Gamma^{+}, \Gamma_{\operatorname{Im}}: H^{+} \rightarrow \operatorname{ker} L^{+}=$ $H^{+} / \operatorname{Im} L_{0}$ and $\Gamma_{\operatorname{Im}}^{+}: H \rightarrow \operatorname{ker} L=H / \operatorname{Im} L_{0}^{+}$, are mutually compatible with certain continuous operators $L_{C}: C(L) \rightarrow \operatorname{ker} L^{+}$and $L_{C}^{+}: C\left(L^{+}\right) \rightarrow \operatorname{ker} L$, that is, $L \hat{\Gamma}=\hat{\Gamma}_{\operatorname{Im}} L_{C}$ and $L^{+} \hat{\Gamma}^{+}=\hat{\Gamma}_{\operatorname{Im}}^{+} L_{C}^{+}$. Let $i: D(L) \rightarrow H, i^{+}: D\left(L^{+}\right) \rightarrow H^{+}$be embeddings. Then

$$
\hat{\mathcal{L}}_{C}=\hat{\Gamma}^{+\prime} i^{+\prime} \hat{\Gamma}_{\operatorname{Im}}^{+} L_{C}-L_{C}^{+\prime} \hat{\Gamma}_{\operatorname{Im}} i \hat{\Gamma}, \quad \hat{\mathcal{L}}_{C}^{+}=\hat{\Gamma}^{\prime} i^{\prime} \hat{\Gamma}_{\operatorname{Im}} L_{C}^{+}-L_{C}^{\prime} \hat{\Gamma}_{\operatorname{Im}}^{+} i^{+} \hat{\Gamma}^{+} .
$$

Proof. For arbitrary $u \in D(L), v \in D\left(L^{+}\right)$we have

$$
\begin{aligned}
\left\langle\hat{\mathcal{L}}_{C} \Gamma u, \Gamma^{+} v\right\rangle & =\left\langle\hat{\mathcal{L}}_{C} \Gamma \hat{\Gamma} \Gamma u, \Gamma^{+} \hat{\Gamma}^{+} \Gamma^{+} v\right\rangle \\
& =\left(L \hat{\Gamma} \Gamma u, i^{+} \hat{\Gamma}^{+} \Gamma^{+} v\right)_{H^{+}}-\left(i \hat{\Gamma} \Gamma u, L^{+} \hat{\Gamma}^{+} \Gamma^{+} v\right)_{H} \\
& =\left(\hat{\Gamma}^{+\prime} i^{+\prime} L \hat{\Gamma} \Gamma u, \Gamma^{+} v\right)_{H^{+}}-\left(\hat{\Gamma}^{+\prime} L^{+\prime} i \hat{\Gamma} \Gamma u, \Gamma^{+} v\right)_{H^{+}} \\
& =\left(\hat{\Gamma}^{+\prime} i^{+\prime} L \hat{\Gamma} \Gamma u-\hat{\Gamma}^{+\prime} L^{+\prime} i \hat{\Gamma} \Gamma, \Gamma^{+} v\right)_{H^{+}} \\
& =\left(\hat{\Gamma}^{+\prime} i^{+\prime} \hat{\Gamma}_{\operatorname{Im}}^{+} L_{C} \Gamma u-L_{C}^{+\prime} \hat{\Gamma}_{\operatorname{Im}} i \hat{\Gamma} \Gamma u, \Gamma^{+} v\right)_{H^{+}},
\end{aligned}
$$

as required.

Consider the form $g\left(\Gamma u, \Gamma^{+} v\right)=\left\langle\mathcal{L}_{C} \Gamma u, \Gamma^{+} v\right\rangle$, which is the restriction of the Green's form $G(u, v)$ to the boundary spaces. The form $g$ brings about a duality between the spaces $C(L)$ and $C\left(L^{+}\right)$(with regard to this and other definitions, which follow below, see [3]) and is nondegenerate. The $g$-polar of the linear space $B \subset C(L)$ is by definition the set $B^{+}$of those $\Gamma^{+} v \in C\left(L^{+}\right)$such that $g\left(\Gamma u, \Gamma^{+} v\right)=0$ for all $\Gamma u \in B$, and the $g$-polar of the linear space $B^{+} \subset C\left(L^{+}\right)$is by definition the set $B$ of those $\Gamma u \in C(L)$ such that $g\left(\Gamma u, \Gamma^{+} v\right)=0$ for all $\Gamma^{+} v \in B^{+}$.

It is not difficult to prove the following properties of $g$-polars, in view of the fact that $\mathcal{L}_{C}$ is an isomorphism; these properties are paraphrases of the analogous properties of orthogonal complements:

1 ) the $g$-polar is closed; the $g$-polar of the $g$-polar of the subspace $B$ (that is, $B^{++}$) is the closure $\bar{B}$ in the space $C(L)$ and, moreover, $\forall B, B^{+++}=B^{+}$;

2 ) the $g$-polar of the sum of two closed subspaces is equal to the intersection of the $g$-polars; the $g$-polar of the intersection of two closed subspaces is equal to the closure of the sum of the $g$-polars;

3 ) if two closed subspaces decompose the space $C(L)$ into a direct sum, then their $g$-polars decompose the space $C\left(L^{+}\right)$into a direct sum.

In this terminology, part 1) of Proposition 2.9 says that the $g$-polar of the space $C\left(\operatorname{ker} L^{+}\right)$coincides with the space $C(\operatorname{ker} L)$. Note that if we introduce the form $g$ by the formula

$$
g\left(\Gamma u, \Gamma^{+} v\right)=G(u, v),
$$


then the proof of part 1) of Proposition 2.9 actually yields the following result.

Proposition 2.11. In order that the g-polar of the subspace $C$ (ker $L^{+}$) coincide with the subspace $C(\operatorname{ker} L)$ it is necessary and sufficient that (1.8) hold. In order that the $g$-polar of the subspace $C(\operatorname{ker} L)$ coincide with the subspace $C\left(\operatorname{ker} L^{+}\right)$it is necessary and sufficient that (1.9) hold.

We now give the definition of the adjoint problem.

Definition 2.4. The boundary-value problem

$$
L^{+} v=h, \quad \Gamma^{+} v \in B^{+}
$$

is called the adjoint of the problem (1.5), if the subspace $B^{+} \subset C\left(L^{+}\right)$is the $g$-polar of the subspace $B \subset C(L)$.

Proposition 2.12. The conditions (1.8), (1.9) hold. The adjoint boundary-value problem (2.18) is correct if and only if the original problem (1.5) is correct.

Proof. Applying Hörmander's work, as problem (1.5) is correct, we have the expansion into a direct sum $C(L)=C(\operatorname{ker} L) \oplus B$. It follows from item 3$)$ of the properties of $g$-polars above that we have the direct-sum expansion $C\left(L^{+}\right)=C\left(\operatorname{ker} L^{+}\right) \oplus B^{+}$, which implies the correctness of problem (2.18).

2.4. The general form of the boundary-value problem. In this subsection we obtain a more explicit representation of the boundary condition for problem (1.5) with closed extension $L_{B}$, by using the existence of $L$-traces and the embedding $A(L) \subset$ $H^{+(-l)}$. Problem (1.5) is then given by a closed subspace $B \subset C(L)$, and, as we showed in Proposition 2.8, the space $C(L)$ is isomorphically mapped by the boundary operator $\mathcal{L}_{C}$ onto the space $C^{*}\left(L^{+}\right)$. The space $C^{*}\left(L^{+}\right)$, which is isomorphic to the space $A(L)$, is embedded in $H^{+(-l)}$ if (1.3), (1.4) hold, and therefore the subspace $B$ is embedded in $H^{+(-l)}$. Let $\bar{B}$ be the closure of the embedded image in $H^{+(-l)}$ and let $\hat{B}: H^{+(-l)} \rightarrow$ $H^{+(-l)}$ be some continuous operator with kernel $\bar{B}$, for example, the projection onto the orthogonal complement of $\bar{B}$ in $H^{+(-l)}$. Then the condition $\hat{B} \mathcal{L}_{C} u=0$ uniquely singles out from the boundary space $C(L)$ the subspace $B$, since the topology in $A(L)$ is stronger than the topology of $H^{+(-l)}$. Next, we introduce in the space $H^{+(-l)}$ the embeddings $j^{+k}: H_{\partial}^{+-k-1 / 2} \rightarrow H^{+(-l)}$ and the projections $q_{k}^{+}: H^{+(-l)} \rightarrow H_{\partial}^{+-k-1 / 2}$ in the direct product. Then the condition $\hat{B} \mathcal{L}_{C} u=0$, which, in view of the equality

$$
\sum_{k=0}^{l} j^{+k} q_{k}^{+}=i d_{H^{+(-l)}}
$$

can be written in the form

$$
\hat{B} L_{\partial} u=\hat{B} \sum_{k=0}^{l} j^{+k} L_{(k)} u=0,
$$

singles out the domain of definition of the extension, $D\left(L_{B}\right)$, in the space $D(L)$. We apply the projection $q_{i}^{+}$to the last equality and set $B_{i}^{+k}:=q_{i}^{+} \hat{B} j^{+k}$. As a result we obtain the equality

$$
\sum_{k=0}^{l} B_{i}^{+k} L_{(k)} u=0 .
$$

We have thus proved the following result. 
Proposition 2.13. Under the conditions (1.3), (1.4), for $u \in D(L)$, each boundary condition $\Gamma u \in B$ with closed subspace $B \subset C(L)$ in (1.5) can be written in the form

$$
B_{i}^{+0} L_{(0)} u+B_{i}^{+1} L_{(1)} u+\cdots+B_{i}^{+l-1} L_{(l-1)} u=0, \quad i=1, \ldots, l,
$$

where the $B_{i}^{+k}: H_{\partial}^{+-k-1 / 2} \rightarrow H_{\partial}^{+-i-1 / 2}$ are continuous operators and, conversely, each condition of the form (2.19), where $B_{i}^{+k}: H_{\partial}^{+-k-1 / 2} \rightarrow H_{\partial}^{+-i-1 / 2}, i, k=1, \ldots, l$, are continuous operators, defines a homogeneous boundary-value problem with closed extension.

We now choose a continuous operator $\hat{B}$ with kernel $\bar{B}$, acting from $H^{+(-l)}$ to $H^{(-l)}$ (above, in Proposition 2.13, $\hat{B}: H^{+(-l)} \rightarrow H^{+(-l)}$ ). We also introduce the projections $q_{k}: H^{(-l)} \rightarrow H_{\partial}^{-k-1 / 2}$ and we apply the projection $q_{i}$ to the equality

$$
\hat{B} L_{\partial} u=\hat{B} \sum_{k=0}^{l} j^{+k} L_{(k)} u=0
$$

in place of the projection $q_{i}^{+}$. Setting $B_{i}^{k}:=q_{i} \hat{B} j^{+k}$, similarly to Proposition 2.13 we obtain

Proposition 2.14. Under the conditions (1.3), (1.4), for $u \in D(L)$, each boundary condition $\Gamma u \in B$ with closed subspace $B \subset C(L)$ in (1.5) can be written in the form

$$
B_{i}^{0} L_{(0)} u+B_{i}^{1} L_{(1)} u+\cdots+B_{i}^{l-1} L_{(l-1)} u=0, \quad i=1, \ldots, l,
$$

where the $B_{i}^{k}: H_{\partial}^{+-k-1 / 2} \rightarrow H_{\partial}^{-i-1 / 2}$ are continuous operators, and conversely, each condition of the form (2.18) with continuous operators $B_{i}^{k}: H_{\partial}^{+-k-1 / 2} \rightarrow H_{\partial}^{-i-1 / 2}$, $i, k=1, \ldots, l$, defines a homogeneous boundary-value problem with closed extension.

\section{Properties of Differential operators in a Domain}

In this section we are in the framework given in paragraphs I and II of $\S 0$. Under the conditions $(0.1),(0.2)$, we obtain a description of the smooth part of the domain of definition of the minimal operator and we apply it to obtain some conditions for the existence of a correct boundary-value problem and a comparison of minimal operators with variable coefficients. We shall consider conditions for the existence of fundamental solutions, the representation of solutions in terms of analogues of classical potentials and the validity of the mean-value theorem.

3.1. Smooth functions from the domain of definition of the minimal operator of a differential operation to a domain with a smooth boundary. In this section we consider a scalar differential operation, that is, we are in the situation described in subsections I, II of $\S 0$, with $N=N^{+}=1$, where for simplicity the domain $\Omega$ is bounded and lies on one side of its smooth boundary $\partial \Omega$. We merely note that for the case $N=N^{+} \neq 1$ the exposition changes only in the appearance of a vector index in the designation of the spaces, and also the appearance of the determinant symbol det in front of the leading symbol $\lambda_{l}(x, \nu(x))$ of the operation $\mathcal{L}$ in the obvious positions.

Throughout this subsection we assume that conditions (1.3), (1.4) hold. We consider the structure of the domain of definition of the minimal operator $L_{0}$ in its smooth part. The next result is a consequence of Proposition 2.5 (or of formula (0.4) and the definitions of minimal and maximal operators).

Proposition 3.1. Under the conditions (1.3), (1.4) the function $u \in H^{l}(\Omega)$ belongs to the space $D\left(L_{0}\right)$ if and only if the traces on the boundary

$\gamma u=\psi=\left(\psi_{0}, \psi_{1}, \ldots, \psi_{l-1}\right) \in H^{(l)}(\partial \Omega):=H^{l-1 / 2}(\partial \Omega) \oplus H^{l-3 / 2}(\partial \Omega) \oplus \cdots \oplus H^{1 / 2}(\partial \Omega)$ 
satisfy the following equation on $\partial \Omega$ :

$$
\mathcal{L}_{\partial \Omega} \gamma u=0,
$$

which in expanded form consists of the following system of equations:

$$
\left\{\begin{array}{r}
(-1)^{l-1} \lambda_{l}(x,-i \nu(x)) \psi_{0}=0 \\
(-1)^{l-2} \lambda_{l}(x,-i \nu(x)) \psi_{1}+L_{(10)} \psi_{0}=0 \\
(-1)^{l-3} \lambda_{l}(x,-i \nu(x)) \psi_{2}+L_{(22)} \psi_{1}+L_{(20)} \psi_{0}=0 \\
\ldots \ldots \ldots \ldots \ldots \ldots \ldots \ldots \ldots \ldots \ldots \ldots \ldots \ldots \ldots \ldots \ldots \ldots \ldots \ldots
\end{array}\right.
$$

where $\lambda_{l}(x, \xi)=\sum_{|\alpha|=l} a_{\alpha}(x) \xi^{\alpha}$ is the leading symbol of the operation $\mathcal{L}$.

Thus, the spaces $X=D\left(L_{0}\right) \cap H^{l}(\Omega), H^{l}(\Omega) / X$ and especially $C(L)$ have a very complicated structure. In particular, the component $\psi_{l-1}$ of the trace $\gamma u$ is arbitrary on int $S_{0}$ if $S_{0}=\left\{x \in \partial \Omega \mid \lambda_{l}(x, \nu(x))=0\right\}$ has a nonzero interior, since it only enters the last equation in the series (3.1) with zero coefficient on $S_{0}$.

Apart from that, this complicated situation becomes very simple when we consider the question of the uniqueness of a solution of the system (3.1). Namely, we have the following result.

Proposition 3.2. Under the conditions (1.3), (1.4) the following five statements are equivalent:

$1)$ the set $S_{0}$ of zeros of $\lambda_{l}(x, \nu(x))$ has an empty interior in $\partial \Omega$ :

$$
\operatorname{int} S_{0}=\varnothing
$$

2) the homogeneous system of equations (3.1) has only the trivial solution in $H^{(l)}(\partial \Omega)$ : that is, $\operatorname{ker} \mathcal{L}_{\partial \Omega}=0$;

3) $D\left(L_{0}\right) \cap H^{l}(\Omega)=H_{0}^{l}(\Omega)$;

4) $\operatorname{ker} \mathcal{L}_{\partial \Omega}^{+}=0$;

5) $D\left(L_{0}^{+}\right) \cap H^{l}(\Omega)=H_{0}^{l}(\Omega)$.

In particular, condition (0.5), which in this case has the form meas $S_{0}=0$, is sufficient for condition 1) and therefore for conditions 2)-5).

Proof. The proof follows from the triangular form of the equations (3.1) for ker $\mathcal{L}_{\partial \Omega}$, of the analogous form of the equation for $\operatorname{ker} \mathcal{L}_{\partial \Omega}^{+}$and the following assertion: in order that the equation $\alpha(x) \psi(x)=0$ with given function $\alpha(x) \in C^{\infty}(\partial \Omega)$ and measurable function $\psi(x)$ should imply that $\psi(x)=0$ almost everywhere, it is necessary and sufficient that $S$, the set of zeros of $\alpha$, have an empty interior. To prove this assertion we note that if the measure meas $S=\mu>0$ and $S$ has an empty interior, that is, each point $s \in S$ is a limiting point of the set $U \backslash S$, then we can, by Luzin's theorem, modify $\psi$ on a set $M_{\epsilon} \subset S$ of measure $\epsilon>0$ so that it becomes continuous, from which it follows that $\psi(x)=0$ on $S \backslash M_{\epsilon}$. The assertion that $\psi(x) \neq 0$ on a set of positive measure $\nu$ now leads to a contradiction, since we can choose $\epsilon<\nu$.

Remark 3.1. We observe that in the case when the coefficients of the operation $\mathcal{L}$ are square-matrix-valued functions, Proposition 3.2 remains true if we replace the function $\lambda_{l}(x, \nu(x))$ by $\operatorname{det} \lambda_{l}(x, \nu(x))$.

We have considered the simplest case when the set $S_{0}$ of characteristic points on $\partial \Omega$ has an empty interior. If, on the other hand, $S_{0}$ has a nonempty interior, then, for example, the inclusion $D\left(L_{0}\right) \cap H^{l}(\Omega) \subset H_{0}^{l-1}(\Omega) \cap H^{l}(\Omega)$ is equivalent to the assertion that the solutions with support in $S_{0}$ of all the first-order equations $L_{(10)} \psi_{0}=0 ; L_{(21)} \psi_{1}=0$; $\ldots ; L_{(l-1 l-2)} \psi_{l-2}=0$ are trivial. 
Example 3.1. Suppose that the original operation $\mathcal{L}$ is scalar, real and its leading part is uniformly elliptic. Then $\forall x, \lambda_{l}(x, \nu(x)) \neq 0$ and, in view of Proposition 3.2, we have $D\left(L_{0}\right) \cap H^{l}(\Omega)=H_{0}^{l}(\Omega)$.

Example 3.2. Suppose that the original operation $\mathcal{L}$ is scalar and its leading part has constant coefficients. Suppose also that the domain $\Omega$ is given by the inequality $P(x)>0$ with real irreducible polynomial $P$ of degree $p>1$, which is nondegenerate on the boundary $\partial \Omega=\{x \mid P(x)=0\}:\left.\nabla P\right|_{P=0} \neq 0$. Then $\nu=-\nabla P /|\nabla P|$, the set $\left\{x \mid \lambda_{l}(\nu(x))=0\right\}$ is algebraic, and therefore if condition 1) in Proposition 3.2 does not hold, then the germs of the algebraic varieties at a point in the interior of $S_{0}$ coincide; therefore the polynomial $\lambda_{l}(\nabla P(x))$ is divisible by the polynomial $P$. In other words, clearly, $\operatorname{ker} \mathcal{L}_{\partial \Omega}=0$ and $D\left(L_{0}\right) \cap H^{l}(\Omega)=H_{0}^{l}(\Omega)$ if $P$ does not divide $\lambda_{l}(\nabla P(x))$; in particular, this is the case if the degree $p$ of the polynomial $P$ does not divide the degree $l$ of the polynomial $\lambda_{l}$ (or the degree $N l$ of the polynomial $\operatorname{det} \lambda_{l}$ when the coefficients are $(N \times N)$-matrix-valued).

3.2. Conditions for the existence of a correct boundary-value problem, comparison of differential operators and coincidence of two maximal operators. We now apply the considerations of $\S 3.1$ to questions of the validity of the conditions for the existence of a correct boundary-value problem and the comparison of differential operators. Here we shall suppose that the operators are induced by a differential operation with $\left(N \times N^{+}\right)$-matrix-valued coefficients and that the domain is bounded with a smooth boundary. Suppose that (1.1), (1.2) hold. In particular, it follows from the Vishik decomposition of the domain of definition of the maximal operator (see Proposition 1.2) that $D\left(L_{0}\right) \cap \operatorname{ker} L=0$. Hence $\left(D\left(L_{0}\right) \cap H^{l}(\Omega)\right) \cap\left(\operatorname{ker} L \cap H^{l}(\Omega)\right)=0$. Therefore we have the following result.

Proposition 3.3. In order that the operator $L_{0}$ have a soluble extension and that $L$ have a correct boundary-value problem (1.5) it is necessary that the traces $\psi$ of any smooth solution (in the space $H^{l}(\Omega)$ ) of the equation $\mathcal{L} u=0$ do not belong to the kernel of the operator $\mathcal{L}_{\partial \Omega}$, that is, they should not belong to the system of equations (3.1), and that the traces of any smooth solution of the equation $\mathcal{L}^{+} u=0$ should not belong to the kernel of the operator $\mathcal{L}_{\partial \Omega}^{+}$.

We recall that one of the definitions (due to Hörmander) of the comparison of two differential operators $\mathcal{L} \prec \mathcal{P}$ with constant coefficients can be stated in the form $D\left(L_{0}\right) \supset$ $D\left(P_{0}\right)$ (43, 45]; see also $\left.\S 1.2\right)$. We shall apply this definition for operators with smooth matrix-valued coefficients. It immediately follows from this that $D\left(L_{0}\right) \cap H^{l}(\Omega) \supset$ $D\left(P_{0}\right) \cap H^{l}(\Omega)$, so that we have the following result.

Proposition 3.4. Suppose that two differential operators $\mathcal{L}$ and $\mathcal{P}$ have the same order $l$ and the same size of matrix-valued coefficients $N \times N^{+}$. In order that $\mathcal{L} \prec \mathcal{P}$ it is necessary that

$$
\operatorname{ker} \mathcal{L}_{\partial \Omega} \supset \operatorname{ker} \mathcal{P}_{\partial \Omega} \text {. }
$$

Hence, recalling the expression for $L_{(0)} u$ (see (3.1)), we obtain the following result.

Proposition 3.5. Under the conditions of Proposition 3.4 with $N=N^{+}$, the relation $\mathcal{L} \succ \mathcal{P}$ holds only if the set of characteristic points

$$
S_{0}(\mathcal{L})=\left\{x \in \partial \Omega \mid \operatorname{det} \lambda_{l}(x, \nu(x))=0\right\}
$$

of the operator $\mathcal{L}$ on the boundary of the domain $\partial \Omega$ is included in the set of characteristic points

$$
S_{0}(\mathcal{P})=\left\{x \in \partial \Omega \mid \operatorname{det} p_{l}(x, \nu(x))=0\right\}
$$

of the operator $\mathcal{P}$ on $\partial \Omega$. 
We now turn to conditions (1.3) and (1.4). Recall that, in accordance with Proposition 2.7, conditions (2.12) and (2.13) suffice for the validity of condition (1.3). But condition (2.12) is equivalent to condition (3.2) by Proposition 3.2 and by Remark 3.1. We therefore have the following result.

Proposition 3.6. Let $N=N^{+}$. In order that (1.3) hold it suffices that conditions (3.2), (2.13) hold. In order that condition (1.4) hold it suffices that (3.2), (2.14) hold.

3.3. Fundamental solutions of differential operators. In this subsection we note the connection between fundamental solutions of the operators $\mathcal{L}$ and $\mathcal{L}^{+}$in $\Omega$, by which we mean distributions $\mathcal{E}_{y} \in D^{\prime}(\Omega)$ and $\mathcal{E}_{y}^{+} \in D^{\prime}(\Omega)$, respectively, satisfying the equations

$$
\mathcal{L}_{x} \mathcal{E}_{y}=\delta(y-x), \quad \mathcal{L}_{x}^{+} \mathcal{E}_{y}^{+}=\delta(y-x), \quad y \in \Omega .
$$

As in $\S 3.1$ above, we shall consider here the case of a scalar differential operation in an arbitrary bounded domain.

Proposition 3.7. If $\mathcal{L}$ has a fundamental solution $\mathcal{E}_{y}(x)$ belonging to the space $L_{2}(\Omega)$ for almost all $y \in \Omega$, then for each $u \in D\left(L_{0}\right)$ and for almost all $y \in \Omega$ one has the representation

$$
u(y)=\left\langle L_{0} u, \mathcal{E}_{y}^{+}\right\rangle .
$$

The proof follows from the equality $\langle\mathcal{L} w, v\rangle=\left\langle w, \mathcal{L}^{+} v\right\rangle$ for any $v \in D^{\prime}(\Omega)$ and any $w \in C_{0}^{\infty}(\Omega)$ by directly substituting $v=\mathcal{E}^{+}$and passing to the limit $w_{j} \rightarrow u$ in the graph norm.

Proposition 3.8. Suppose that $\mathcal{L}^{+}$has a fundamental solution $\mathcal{E}_{y}^{+} \in L_{2}(\Omega)$ and suppose that either (1.8) holds for the normal solubility of the operator $L_{0}$, or $\mathcal{E}^{+} \in L_{2}(\Omega \times \Omega)$. Then condition (1.1) holds.

Indeed, it follows from (3.3) that the kernel, ker $L_{0}$, is trivial, and it follows from condition (1.8) and Banach's inverse-operator theorem that the left inverse of the operator $L_{0}$ given by (3.3) is continuous. Suppose now that $\mathcal{E}_{y}^{+} \in L_{2}(\Omega \times \Omega)$ and that $u_{k} \in$ $D\left(L_{0}\right)$ is a sequence such that $L_{0} u_{k} \rightarrow U$ in $L_{2}(\Omega)$. Then it follows from (3.3) that $\left|u_{k}(y)-u_{m}(y)\right|^{2} \leq\left\|L_{0} u_{k}-L_{0} u_{m}\right\|^{2}\left\|\mathcal{E}_{y}^{+}\right\|^{2}$. Applying Fubini's theorem we see that $u_{k}$ converges in $L_{2}(\Omega)$ to some $u \in D\left(L_{0}\right)$. Since $L_{0}$ is a closed operator, $u \in D\left(L_{0}\right)$, and so condition (1.8) holds, which together with the equality $\operatorname{ker} L_{0}=0$ yields condition (1.1).

Proposition 3.9. Suppose that condition (1.1) holds and each function in $D\left(L_{0}\right)$ is continuous in the domain $\Omega$ perhaps after correcting it on a set of measure zero. Then $\mathcal{L}^{+}$has a fundamental solution $\mathcal{E}_{y}^{+}$lying in $L_{2}(\Omega)$; furthermore, the map $\mathcal{E}^{+}: \Omega \rightarrow L_{2}(\Omega)$ acting by the formula $y \rightarrow \mathcal{E}_{y}^{+}$is weakly continuous in the space $\operatorname{Im} L_{0}$.

Proof. It follows from the existence of the left inverse $M$ :

$$
M L_{0}=\left.\mathrm{id}\right|_{D\left(L_{0}\right)}
$$

that the right inverse $M_{1}$ exists:

$$
\mathcal{L}^{+} M_{1}=\left.\mathrm{id}\right|_{D^{\prime}\left(L_{0}\right)}
$$

with $L_{2}(\Omega)$-conjugate space $D^{\prime}\left(L_{0}\right)$, which implies the map $\mathcal{L}^{+}: L_{2}(\Omega) \rightarrow D^{\prime}\left(L_{0}\right)$ is surjective. It follows from the embedding $D\left(L_{0}\right) \subset C(\Omega)$ that $C^{\prime}(\Omega) \subset D^{\prime}\left(L_{0}\right)$, and since $\delta_{y} \in C^{\prime}(\Omega)$, we obtain the existence of a solution $\mathcal{E}_{y}^{+}$of the equation $\mathcal{L}^{+} \mathcal{E}_{y}^{+}=\delta_{y}$ lying in the space $L_{2}(\Omega)$. Its continuity on $\operatorname{Im} L_{0}$ in the weak topology of $L_{2}(\Omega)$ follows from (3.3) and the continuity of the function $u(y)$. 
Proposition 3.10. Suppose that the operator $\mathcal{L}^{+}$has a fundamental solution $\mathcal{E}_{y}^{+}$belonging to the space $L_{2}(\Omega)$ and weakly continuous in $y \in \Omega$ on the space $\operatorname{Im} L_{0} \subseteq L_{2}(\Omega)$. Then each function in $D\left(L_{0}\right)$ is continuous in $\Omega$.

Proof. This follows from (3.3).

Proposition 3.11. An operator of constant force of the form (1.11) with coefficients that are analytic in $\Omega^{\prime} \supset \bar{\Omega}$ has a fundamental solution in the domain $\Omega$ if the function

$$
\left(\tilde{P}_{0}\right)^{-1}=\left(\sum_{\alpha \leq l}\left|D_{\xi}^{\alpha} P_{0}(\xi)\right|^{2}\right)^{-1 / 2}
$$

belongs to the space $L_{2}\left(\mathbb{R}^{n}\right)$.

Proof. This follows from Propositions 3.9, 1.9 and Theorem 10.1.25 in [43].

Proposition 3.12. An operator of real principal type of the form (1.11), where the $P_{j}$ are operators of order less than $l=\operatorname{deg} P_{0}$, has a fundamental solution if $l>n / 2+1$.

Proof. This follows from Propositions 3.9, 1.10 and Theorem 10.1.25 in 43, and also from the estimate of a solution of an equation of principal type in [44].

3.4. Representation of solutions and the mean value theorem. Here our aim is to obtain a representation of a solution in terms of an analogue of classical potentials. Formula (3.3) is an example of such a representation. As in the previous subsection, we consider the case of a scalar differential operation in a bounded domain, but this time with a smooth boundary.

Proposition 3.13. Suppose that at each point $y \in \Omega$ the operator $\mathcal{L}^{+}$has a fundamental solution $\mathcal{E}_{y}^{+}$, representable in the form $\mathcal{E}_{y}^{+}=e_{y}^{0}+e_{y}^{\infty}$, where $e_{y}^{0} \in \mathcal{E}^{\prime}(\Omega), e_{y}^{\infty} \in C^{\infty}(\bar{\Omega})$. Then each function $u \in C^{\infty}(\bar{\Omega})$ has the representation:

$$
\text { for all } y \in \Omega, \quad u(y)=\left\langle L u, \mathcal{E}_{y}^{+}\right\rangle_{\Omega}+\sum_{q=0}^{l-1}\left\langle L_{(l-q-1)} u, \partial_{\nu}^{q} \mathcal{E}_{y}^{+}\right\rangle_{L_{2}(\partial \Omega)} .
$$

Proof. This follows from Green's formula (0.3). We obtain (3.4) by replacing $v$ in (0.3) by the sequence of functions $v_{k}=e_{y}^{\infty}+\psi_{k}$, where $C_{0}^{\infty}(\Omega) \ni \psi_{k} \rightarrow e_{y}^{0}$ in the space $\mathcal{E}^{\prime}(\Omega)$.

Proposition 3.14. Suppose that under the conditions of Proposition 3.13 the fundamental solution $\mathcal{E}_{y}^{+}$belongs to the space $L_{2}(\Omega)$, that is, $e_{y}^{0} \in L_{2}\left(\Omega_{x}\right)$, supp $e_{y}^{0} \subset \Omega$. Then for each function $u \in D(\tilde{L})$, for almost all $y \in \Omega$, (3.4) holds.

Proof. This follows from Green's formula (2.7). We obtain (3.4) by replacing $v$ in (2.7) by the sequence of functions $v_{k}=e_{y}^{\infty}+\psi_{k}$, where $C_{0}^{\infty}(\Omega) \ni \psi_{k} \rightarrow e_{y}^{0}$ in the space $L_{2}(\Omega)$.

Note that the conditions of Proposition 3.13 hold, for example, for a hypoelliptic operator with constant coefficients [43. We also note that the terms in the representation (3.4) are analogues of the classical potentials, and a formula of type (3.4) is well known for elliptic equations. Finally, we note that in Agranovich's survey [1], similar representations of solutions of equations with constant coefficients can be found.

The next result (a mean value theorem) is an immediate consequence of Proposition 3.14. 
Proposition 3.15. Suppose that under the conditions of Proposition 3.14, where $\left.e_{y}^{\infty}\right|_{\partial \Omega} \in$ $H^{1}(\partial \Omega)$ and $\operatorname{deg} \mathcal{L}=2, u \in C(\bar{\Omega})$ is a solution of the equation Lu=0 in the domain $\Omega$ and suppose that $\mathcal{E}_{y}^{+}(x)=0$ for $x \in \partial \Omega$. Then

$$
u(y)=\left\langle\lambda_{2}(x, \nu(x)) u, \partial_{\nu} \mathcal{E}_{y}^{+}\right\rangle_{\partial \Omega},
$$

where, as before, $\lambda_{2}(x, \xi)$ is the leading symbol of the operator $L$.

Example 3.3. For the Laplace operator with the choice of the fundamental solution

$$
\mathcal{E}_{y}^{+}=\frac{1}{\sigma_{n}}\left[-\frac{1}{n-2}|x-y|^{2-n}+\mathcal{E}_{0}(x)\right] \text { for } n>2
$$

and

$$
\mathcal{E}_{y}^{+}=\frac{1}{2 \pi}\left[-\ln |x-y|+\mathcal{E}_{0}(x)\right] \text { for } n=2
$$

with arbitrary harmonic function $\mathcal{E}_{0}(x)$ chosen such that $\mathcal{E}_{0}(x)=$ const, the last formula becomes the mean value theorem for harmonic functions over spheres or circles. For other functions $\mathcal{E}_{0}(x)$ we get nonstandard mean value theorems for harmonic functions.

Here is a further illustration relating to the heat equation. As is well known, the function

$$
\mathcal{E}_{C}^{+}=\frac{\theta t}{2 \sqrt{\pi t}} e^{-\frac{x^{2}}{(4 t)}}-C
$$

with constant $C$ is a fundamental solution of the hypoelliptic operator $\partial_{t}-\partial_{x}^{2}$. The curve $S_{C}$ defined by the equation $x^{2}+2 t\left(\ln t+C_{0}\right)=0, C_{0}=4 \pi C^{2}$ is the level curve $\mathcal{E}^{+}=0$ for $C>0$. It is a closed infinitely smooth curve that is tangent to the $x$-axis at the origin. We claim that for each solution $u \in C\left(\Omega^{\prime}\right)$ of the equation $\left(\partial_{t}+\partial_{x}^{2}\right) u=0$ we have

$$
u(0)=-\int_{S_{C}} \nu_{2}^{2} u(x) \partial_{\nu} \mathcal{E}_{C}^{+}(x) d s,
$$

where the domain $\Omega$ is bounded by the curve $S_{C}$ and the domain $\Omega^{\prime}$ contains $\bar{\Omega}$. For the proof we include the curve $S_{C}$ in a family of curves encircling the origin that are smooth (with respect to $r$ ); we then apply formula (3.5) and pass to the limit with respect to $r$. Formulae similar to the last one are well known and are used in the theory of parabolic equations and systems (see, for example, [28]).

\section{MAXIMAL EXTENSIONS AND LINEAR BOUNDARY-VALUE PROBLEMS}

In this section we consider realizations of spaces associated with traces that are at the same time boundary spaces for the formally adjoint operation, and we consider an algorithm for solving the boundary-value problem (1.5). We assume that the hypotheses of subsection I of $\S 0$ hold.

4.1. The spaces $A$ and $A^{+}$. We consider the subspace $A:=D_{\perp}(L)$ of $D(L)$ orthogonal to $D\left(L_{0}\right)$ along with the orthogonal projection $P_{A}: D(L) \rightarrow A$ onto it; similarly, we consider the subspace $A^{+}:=D_{\perp}\left(L^{+}\right)$of $D\left(L^{+}\right)$, orthogonal to $D\left(L_{0}^{+}\right)$along with the orthogonal projection $P_{A^{+}}: D\left(L^{+}\right) \rightarrow A^{+}$. Recall that in Proposition 2.8 the space of associated traces $A(L)$ was identified with the conjugate space $C^{*}\left(L^{+}\right)$, which is isomorphic to the orthogonal complement $A^{+}$of $D\left(L_{0}^{+}\right)$in $D\left(L^{+}\right)$. This enables us to consider $A^{+}$to be the space of associated traces $A(L)$, and the space $A$ to be the space of associated traces $A\left(L^{+}\right)$. Furthermore, in the Hilbert case considered by us, $A^{+}$can also be considered to be a realization of the boundary space $C\left(L^{+}\right)$. Similarly, $A$ can also be considered to be a realization of the boundary space $C(L)$. In what follows we set $L_{A}:=\left.L\right|_{A}$ and $L_{A^{+}}^{+}:=\left.L^{+}\right|_{A^{+}}$. 
We denote by $\mathcal{L}^{\prime}, \mathcal{L}^{+\prime}$ the dual operators of the original operators:

$$
\mathcal{L}: H_{0}^{l} \rightarrow H^{+}, \quad \mathcal{L}^{+}: H_{0}^{+l} \rightarrow H, \quad\left(\mathcal{L}^{+}\right)^{\prime}: H^{+} \rightarrow\left(H_{0}^{l}\right)^{\prime}, \quad \mathcal{L}^{\prime}: H \rightarrow\left(H_{0}^{+l}\right)^{\prime},
$$

where we assume, in view of the equality in item $\mathrm{I}_{2}$ of $\S 0$, that the operators $\left.\mathcal{L}\right|_{H_{0}^{l}}$ and $\left.\mathcal{L}^{+}\right|_{H_{0}^{+l}}$ (as well as the operators $L$ and $L^{+}$) are the restrictions: $\mathcal{L}=\left.\mathcal{L}^{+\prime}\right|_{H_{0}^{l}}$ and $\mathcal{L}^{+}=\left.\mathcal{L}^{\prime}\right|_{H_{0}^{+l}}$.

\section{Proposition 4.1.}

1) The operators $L_{A}: A \rightarrow A^{+}$and $L_{A^{+}}^{+}: A^{+} \rightarrow A$ are isometrically isomorphic and $L_{A^{+}}^{+} L_{A}=-i d_{A}, L_{A} L_{A^{+}}^{+}=-i d_{A^{+}}$.

2) The space $A$ coincides with the kernel of the operator

$$
\mathcal{L}^{\prime} \mathcal{L}^{+\prime}+\left.1\right|_{D(L)}=L^{\prime} L+i^{\prime} i: D(L) \rightarrow D^{\prime}(L),
$$

where $i: D(L) \subset H$ and the operators $i^{\prime}$ and $L^{\prime}: H^{+} \rightarrow D^{\prime}(L)$ are dual in the rigged spaces. The space $A^{+}$coincides with the kernel of the operator $\mathcal{L}^{+\prime} \mathcal{L}^{\prime}+\left.1\right|_{D\left(L^{+}\right)}$in $D\left(L^{+}\right)$.

3) For $u$ in $D(L)$ and $v$ in $D\left(L^{+}\right)$the Green's form is calculated by the formula

$$
\begin{gathered}
G(u, v)=(L u, v)_{H^{+}}-\left(u, L^{+} v\right)_{H}=\left(L P_{A} u, P_{A^{+}} v\right)_{D\left(L^{+}\right)}=-\left(P_{A} u, L^{+} P_{A^{+}} v\right)_{D(L)} ; \\
\text { in particular, for } u \in A \text { and } v \in A^{+} \text {we have } G(u, v)=(L u, v)_{A^{+}}=-\left(u, L^{+} v\right)_{A} .
\end{gathered}
$$

Proof. Item 1) is a rewording (for convenience) of our notation in part 1) of Proposition 1.7 .

2) The inclusion $\left.A \subset \operatorname{ker}\left(\mathcal{L}^{\prime} \mathcal{L}^{+\prime}+1\right)\right|_{D(L)}$ follows from item 1). It is clear from the definitions that $\left.\mathcal{L}^{+\prime}\right|_{D(L)}=L$. Conversely, if $u \in D(L)$ and $\mathcal{L}^{\prime} L u=-u$, then for any $w \in H_{0}^{l}$ we have $-\langle u, w\rangle=\langle L u, \mathcal{L} w\rangle$, that is, $(u, w)_{D(L)}=0$; similarly $v \in D\left(L^{+}\right)$and $\mathcal{L} L^{+} v=-v$ imply that for any $w \in H_{0}^{+l}$ we have $(v, w)_{D\left(L^{+}\right)}=0$.

3) For each $u \in A$ and $v \in A^{+}$we have

$$
(L u, v)_{D\left(L^{+}\right)}=(L u, v)_{H^{+}}+\left(L^{+} L u, L^{+} v\right)_{H}=(L u, v)_{H^{+}}-\left(u, L^{+} v\right)_{H}=G(u, v) .
$$

If

$$
\begin{array}{cc}
D(L) \ni u=u_{0}+u_{A}, & u_{0} \in D\left(L_{0}\right), \quad u_{A} \in A, \\
D\left(L^{+}\right) \ni v=v_{0}+v_{A^{+}}, & v_{0} \in D\left(L_{0}^{+}\right), \quad v_{A^{+}} \in A^{+},
\end{array}
$$

then for the Green's form we obtain

$$
\begin{aligned}
G(u, v) & =\left(L u_{0}+L u_{A}, v_{0}+v_{A^{+}}\right)-\left(u_{0}+u_{A}, L^{+} v_{0}+L^{+} v_{A^{+}}\right) \\
& =\left(L u_{0}, v_{0}\right)+\left(L u_{A}, v_{A^{+}}\right)-\left(u_{0}, L^{+} v_{0}\right)-\left(u_{A}, L^{+} v_{A^{+}}\right) \\
& =\left(L u_{A}, v_{A^{+}}\right)-\left(u_{A}, L^{+} v_{A^{+}}\right)=G\left(u_{A}, v_{A}\right) \\
& =\left(L u_{A}, v_{A^{+}}\right)_{D\left(L^{+}\right)}=-\left(u_{A}, L^{+} v_{A^{+}}\right)_{D(L)} .
\end{aligned}
$$

This completes the proof.

Note that any subspace $E$ of $D(L)$ containing $D\left(L_{0}\right)$ has a nonzero intersection (and projection) $E \cap A=P_{A} E$ and is determined by it. In particular, the subspace $D(\tilde{L})$ is determined by its projection $\tilde{A}:=A \cap D(\tilde{L})$, the subspace $\operatorname{ker} L$ is determined by the projection $K:=A \cap \operatorname{ker} L$, and the subspace $D\left(L_{B}\right)$ defining the extension and the boundary-value problem is determined by its projection $B:=P_{A} D\left(L_{B}\right)=A \cap D\left(L_{B}\right)$. Here we shall take the liberty of denoting by the same letter $B$ subspaces of (in general) different spaces $A$ and $C(L)$, motivated by the fact that the first realizes the second. The spaces $\tilde{A}^{+}, K^{+}, B^{+}$of $A^{+}$are defined similarly. 


\section{Proposition 4.2.}

1) Under conditions (1.1), (1.2) the projections $K=P_{A} \operatorname{ker} L, K^{+}=P_{A^{+}} \operatorname{ker} L^{+}$ are closed.

2) The spaces $K$ and $L_{A^{+}}^{+} K^{+}$are orthogonal in $A$, and the subspaces $K^{+}$and $L_{A} K$ are orthogonal in $A^{+}$.

3) The subspaces $B$ and $L_{A^{+}}^{+} B^{+}$are orthogonal in $A$, and the subspaces $B^{+}$and $L_{A} B$ are orthogonal in $A^{+}$.

Proof. Assertion 1) follows from assertion 1) of Proposition 1.2. The proof of assertion 2) follows from assertion 3) of Proposition 4.1 (cf. Proposition 2.9). The proof of assertion 3) follows from Definition 2.4 in $\S 2.3$.

We now wish to single out conditions enabling us to regard the topology induced in the space $A$ from $D(L)$ as equivalent to the topology induced from $H$. We consider two conditions:

$$
\begin{aligned}
& \forall u \in A, \quad\|L u\|_{H^{+}} \geq c_{1}\|u\|_{H}, \\
& \forall v \in A^{+}, \quad\left\|L^{+} v\right\|_{H} \geq c_{2}\|v\|_{H^{+}} .
\end{aligned}
$$

Note that, in view of the equality $L_{A} L_{A^{+}}^{+} v=-v$, condition (4.1) is equivalent to the condition $\forall v \in A^{+},\|v\|_{H^{+}} \geq c_{1}\|L v\|_{H}$, and this means the same as the assertion that the norms of $D\left(L^{+}\right)$and $H^{+}$are equivalent in $A^{+}$:

$$
\forall v \in A^{+}, \quad\|v\|_{H^{+}} \geq\|v\|_{D\left(L^{+}\right)} \geq\left(1+c_{1}\right)\|v\|_{H^{+}}
$$

and a similar argument prevails for condition (4.2). We have proved the following result.

\section{Proposition 4.3.}

1) The norms of $D(L)$ and $H$ in $A$ are equivalent if and only if condition (4.2) holds.

2) The norms of $D\left(L^{+}\right)$and $H^{+}$are equivalent in $A^{+}$if and only if condition (4.1) holds.

These conditions also enable us to give certain sufficient conditions for (1.3) and (1.4) to hold; see Remark 4.4 below. In particular, for a scalar differential equation with constant coefficients in a bounded domain, condition (4.2) is sufficient for condition (1.3) to hold, and condition (4.1) is sufficient for condition (1.4) to hold.

Remark 4.1. The verification of conditions (4.1), (4.2) for concrete operators can be made easier if one knows some extra information about the structure of the spaces $A$ and $A^{+}$. For example, suppose that the operation $\mathcal{L}$ is formally selfadjoint. Following von Neumann's scheme we introduce the deficiency subspaces $A_{+}:=\operatorname{ker}\left(\mathcal{L}^{\prime}-i\right) \cap D(L)$ and $A_{-}:=\operatorname{ker}\left(\mathcal{L}^{\prime}+i\right) \cap D(L)$. We then have the orthogonal decomposition $A=A_{+} \oplus A_{-}$ (see [22], §XII.4). We consider the example of a formally selfadjoint scalar differential operation with constant real coefficients in an arbitrary bounded domain that is formally selfadjoint. Note that the spaces $A_{+}$and $A_{-}$, which are subspaces of eigenfunctions, have a topology equivalent to that induced from the space $H$. However, in spite of the fact that they are orthogonal in $A$, their sum is not necessarily embedded in $H$ as a closed subspace and does not have the same topology as $H$. In order for this to happen it is necessary and sufficient that the angle between them in $H$ be greater than zero, that is, in order that conditions (4.1) and (4.2) (which coincide with each other) hold, it is necessary and sufficient that

$$
\exists \varepsilon>0, \quad \forall u_{+} \in A_{+}, \quad \forall u_{-} \in A_{-}, \quad \frac{\left|\left(u_{+}, u_{-}\right)_{H}\right|}{\left\|u_{+}\right\|_{H}\left\|u_{-}\right\|_{H}}<1-\varepsilon .
$$


Making condition (4.3) explicit, we obtain the next result.

\section{Proposition 4.4.}

1) For a scalar formally selfadjoint differential operation $\mathcal{L}$ with constant coefficients and full symbol $\tilde{\lambda}(\xi)$ in an arbitrary bounded domain $\Omega$, condition (4.3) implies the following condition:

$$
\exists \varepsilon>0, \forall \xi \in \Lambda_{i}, \forall \eta \in \Lambda_{i}, \quad \frac{\left|\widehat{\theta}_{\Omega}(\xi-\eta)\right|^{2}}{\widehat{\theta}_{\Omega}(2 i \operatorname{Im} \xi) \widehat{\theta}_{\Omega}(-2 i \operatorname{Im} \eta)}<1-\varepsilon .
$$

Here $\Lambda_{ \pm i}=\left\{\xi \in \mathbb{C}^{n} \mid \tilde{\lambda}(\xi)= \pm i\right\}$ is the algebraic variety of zeros of the symbol $\tilde{\lambda}(\xi) \mp i$ of the operation $\mathcal{L}^{\prime} \mp i$, and $\widehat{\theta}_{\Omega}$ is the Fourier transform of the characteristic function $\theta_{\Omega}$ of the domain $\Omega$.

2) If in the kernel of the operator $\operatorname{ker}\left(\mathcal{L}^{\prime} \mathcal{L}^{+\prime}+1\right)$ in the space $L_{2}(\Omega)$ the exponential solutions $e^{i(\xi, x)}, \xi \in \Lambda_{ \pm} i$, are dense, then condition (4.4) implies condition (4.3).

Proof. Since the differential operation $\mathcal{L}$ is formally selfadjoint, thus $\overline{\tilde{\lambda}(\xi)}=\tilde{\lambda}(\bar{\xi})$. We choose the solutions $u_{+}$of the equation $L u_{+}=i u_{+}$in the form $u_{+}=e^{i(\xi, x)}, \xi \in \Lambda_{i}$, while the solutions $u_{-}$of the equation $L u_{-}=-i u_{-}$are the functions $u_{-}=e^{i(\bar{\xi}, x)}, \xi \in \Lambda_{i}$.

The left-hand side of (4.3) becomes:

$$
\begin{aligned}
\forall \xi, \eta \in \Lambda_{i}, \quad \frac{\left|\left(u_{+}, u_{-}\right)_{H}\right|^{2}}{\left\|u_{+}\right\|_{H}^{2}\left\|u_{-}\right\|_{H}^{2}} & =\frac{\left|\int_{\Omega} e^{i(\xi, x)} \overline{e^{i(\bar{\eta}, x)}} d x\right|^{2}}{\left(\int_{\Omega} e^{i(\xi, x)} e^{-i(\bar{\xi}, x)} d x\right)\left(\int_{\Omega} e^{i(\bar{\eta}, x)} e^{-i(\eta, x)} d x\right)} \\
& =\frac{\left|\int_{\Omega} e^{i(\xi-\eta, x)} d x\right|^{2}}{\left(\int_{\Omega} e^{-2(\operatorname{Im} \xi, x)} d x\right)\left(\int_{\Omega} e^{2(\operatorname{Im} \eta, x)} d x\right)} \\
& =\frac{\left|\widehat{\theta}_{\Omega}(\xi-\eta)\right|^{2}}{\widehat{\theta}_{\Omega}(2 i \operatorname{Im} \xi) \widehat{\theta}_{\Omega}(-2 i \operatorname{Im} \eta)} .
\end{aligned}
$$

4.2. Boundary-value problems. In this subsection we consider boundary-value problems for the equation $L u=f$. But before this we set out certain facts touching on generalized settings of boundary-value problems for an equation of the form

$$
\mathcal{L}^{+} \mathcal{L} u=f,
$$

which we shall need below [10, 47.

By a generalized solution of the Dirichlet problem for the equation (4.5) with righthand side $f \in D^{\prime}\left(L_{0}\right)$ we mean an element $u \in D\left(L_{0}\right)$ satisfying the following "integral" identity for any element $v \in H_{0}^{l}$ with minimal operator $L_{0}$ :

$$
\left\langle L_{0} u, \mathcal{L} v\right\rangle_{H^{+}}=\langle f, v\rangle_{H} .
$$

The identity (4.6) is clearly equivalent to the identity

$$
\left\langle L_{0} u, L_{0} v\right\rangle_{H^{+}}=\langle f, v\rangle_{H} \quad \forall v \in D\left(L_{0}\right),
$$

which we can write as the equation $L_{0}^{\prime} L_{0} u=f$, where $L_{0}^{\prime}: H^{+} \rightarrow D^{\prime}\left(L_{0}\right)$ is the dual operator (in the sense of rigged spaces) of the operator $L_{0}: D\left(L_{0}\right) \rightarrow H^{+}$.

We say that the generalized Dirichlet problem (4.6) is well-posed or simply correct if for each element $f \in D^{\prime}\left(L_{0}\right)$ there exists a unique generalized solution $u \in D\left(L_{0}\right)$, continuously dependent on $f$, that is, if the operator $L_{0}^{\prime} L_{0}: D\left(L_{0}\right) \rightarrow D^{\prime}\left(L_{0}\right)$ has a continuous two-sided inverse. 
Proposition 4.5. In order that the generalized Dirichlet problem (4.6) be correct it is necessary and sufficient that Vishik's condition (1.1) in $§ 1.1$ hold.

The proof can be found in [47].

Example 4.1. In $\S 1.2$ we listed several classes of differential operators for which conditions (1.1), (1.2) in $\S 1.1$ hold in a bounded domain. We recall that in this list were

i) scalar operators with constant coefficients;

ii) scalar operators of principal type;

iii) scalar operators of constant strength;

iv) matrix-valued operators with constant complex coefficients with the PaneahFuglede property;

v) matrix-valued operators that are uniformly elliptic in the Douglis-Nirenberg sense in a domain with a smooth boundary.

The next result is a consequence of Proposition 4.5.

Proposition 4.6. If $\mathcal{L}$ is one of the operators in the classes $(i)-(v)$ in a bounded domain, then the generalized Dirichlet problem (4.6) for equation (4.5) is correct.

Remark 4.2. The generalized Dirichlet problem (4.6) helps one to find the form of the orthogonal projection $\Gamma_{\operatorname{Im}}: H^{+} \rightarrow \operatorname{ker} L^{+}$and the projection $P_{0}=i_{0}^{-1}: D(L) \rightarrow D\left(L_{0}\right)$ in the Vishik decomposition in part 1) of Proposition 1.2. Namely, under condition (1.1) we define $v=P_{0} u$ for a given $u \in D(L)$ as the solution of the generalized Dirichlet problem for equation (4.5) of the form $L_{0}^{\prime} L_{0} v=L_{0}^{\prime} L u$. The kernel of $L_{0}^{\prime}$ is the same as $\operatorname{ker} L^{+}$; therefore $L_{0}^{\prime} L u=0$ for $u \in \operatorname{ker} L+W$, where $L W=\operatorname{ker} L^{+}$, and $P_{0}^{2}=P_{0}$ on $D\left(L_{0}\right)$. Furthermore, under condition (1.1) the map $U \in H^{+} \rightarrow P_{\operatorname{Im}} U$, where $P_{\operatorname{Im}} U=$ $L_{0} u$ and $u$ is the solution of the generalized Dirichlet problem for equation (4.5) of the form $L_{0}^{\prime} L_{0} u=L_{0}^{\prime} U$, is the orthogonal projection onto $\operatorname{Im} L_{0}$ and $\Gamma_{\operatorname{Im}}=1-P_{\operatorname{Im}}$. This observation enables us to give a sufficient condition for (2.16), which concerns the density of the smooth kernel in ker $L^{+}$in the topology of $H^{+}$, to hold. Suppose that the following condition holds:

there exists a set $\mathcal{E}$ that is dense in $H^{+l}$ such that $P_{\operatorname{Im}} \mathcal{E} \subset H^{+l}$.

Then condition (2.16) holds. Indeed, we then have that the set $\mathcal{E}$ is dense in $H^{+}$, and $\left(1-P_{\mathrm{Im}}\right) \mathcal{E} \subset H^{+l}$ is dense in $\operatorname{ker} L^{+}$.

Remark 4.3. The generalized Dirichlet problem (4.6) enables us to find the form of the projection $P_{A}$ in $\S 4$.1. Consider an operation of the form $\mathcal{M} u=(\underset{\mathcal{L} u}{\mathcal{L} u})$ on the smooth space $H^{l}$. Then $\mathcal{M}^{+}\left(\begin{array}{c}v_{1} \\ v_{2}\end{array}\right)=\mathcal{L}^{+} v_{1}+v_{2}, D(M)=D(L), D\left(M_{0}\right)=D\left(L_{0}\right)$. For this operation, condition (1.1) holds as in Example 4.3, and by Proposition 4.5 the Dirichlet problem (4.6) with operator $M$ is correct. The operator $1-P_{A}$, which acts according to the rule $D(L) \ni u \rightarrow v$, where $v$ is the solution of the Dirichlet problem for equation (4.5) of the form $M_{0}^{\prime} M_{0} v=\left(\mathcal{L}^{+} L+1\right) u\left(=\mathcal{M}^{\prime} M u\right)$, is the orthogonal projection with kernel $A$ from $D(L)$ onto $D\left(L_{0}\right)$.

Remark 4.4. The generalized problem (4.6) enables us to find sufficient conditions for the main conditions (1.3), (1.4) to hold. By considering the Dirichlet problem for the equation $\mathcal{R}^{+} \mathcal{R} v=\mathcal{R}^{+} \mathcal{R} u$ with operation $\mathcal{R}=\mathcal{L L}^{+}+1$ we can, in exactly the same way as in Remark 4.2, introduce the condition

$$
\text { there exists a set } \mathcal{E} \text {, dense in } H^{+2 l} \text {, such that } P_{R \operatorname{Im}} \mathcal{E} \subset H^{+2 l}
$$

with initial smooth space $H^{+2 l}=\left[H^{2 l}(\partial \Omega)\right]^{N^{+}} \subset H^{+l}$ for the operation $\mathcal{R}$. This condition means that the smooth space in the kernel ker $R$ is dense with the topology 
of $H^{+}$. This together with condition (4.1) implies that: 1) the space $H^{+2 l}$ is dense in the space $A^{+}$in the topology of $D\left(L^{+}\right), 2$ ) condition (1.4) in $\S 1$ holds since the smooth compactly supported elements are dense in $\left.D\left(L_{0}\right), 3\right)$ condition (1.3) in $\S 1$ holds if we assume that $\mathcal{L}^{+}\left(H^{+2 l}\right) \subset H^{+l}$ since $L^{+} A^{+}=A$. Note that if $\mathcal{L}$ is a scalar differential operator with constant coefficients, the above condition holds in any bounded domain if we take $\mathcal{E}=C^{\infty}(\bar{\Omega})$. Indeed, let $m \in \mathbb{Z}_{+}$be any nonnegative integer and take the spaces in subsection I in $\S 0$ :

$$
H=H^{+}=H^{m}(\Omega), \quad H^{2 l}=H^{+2 l}=H^{m+2 l}(\Omega), \quad H_{0}^{2 l}=H_{0}^{+2 l}=H^{m+2 l}(\Omega) \cap H_{0}^{2 l}(\Omega),
$$

and the operation $\mathcal{R}$ of order $2 l$ defined as above with the help of the operation $\mathcal{L}$ of order $l$. All the conditions in subsection I of $\S 0$ hold, and we can construct a theory of extensions and boundary-value problems as in $\S 1.1$, as well as the theory of the Dirichlet problem in its generalized setting, which is the concern in the current subsection, and which is correct if Vishik's condition (1.1) holds. This condition (1.1) is equivalent to Hörmander's estimate in $\S 1.4$, which in our case follows from the inequality

$$
\left\|\operatorname{grad}^{m} \mathcal{R} \phi\right\|_{L_{2}(\Omega)} \geq C\left\|\operatorname{grad}^{m} \phi\right\|_{L_{2}(\Omega)} \quad \forall \phi \in C_{0}^{\infty}(\Omega),
$$

and, in view of the commutativity of grad and $\mathcal{R}$, this last inequality follows from Hörmander's estimate for the operator $\mathcal{R} D^{\alpha},|\alpha|=m$, in a bounded domain (see $\S 1.2$ ). Our assertion is proved. Hence, in particular, it follows that for an operator with constant coefficients in any bounded domain, condition (1.3), which concerns the density of smooth functions in $D(L)$, holds provided that condition (4.2), concerning the coincidence of the topology of the spaces $D(L)$ and $H$ on the space $A$, holds.

We now turn to boundary conditions of the form (1.5). The boundary-value problem (1.5) gives and is given by a subspace $B \in A$, and the basic questions of the theory of boundary-value problems are questions dealing with the properties of boundary-value problems, including how to find the kernel and cokernel of the operator $L_{B}$ of the boundary-value problem, how to single out correct boundary-value problems, as well as the main question of how to solve the boundary-value problem. The properties of the spaces $A$ and $A^{+}$along with the previous constructions of this subsection enable one to give answers to these questions in terms of subspaces of the spaces $A$ and $A^{+}$and procedures for solving the generalized Dirichlet problem for suitable equations.

We can prove the following.

\section{Proposition 4.7.}

1) The kernel of $L_{B}$ is characterized by the intersection $B \cap K$ :

$$
\operatorname{ker} L_{B}=\operatorname{ker} L \cap D\left(L_{B}\right)=\left\{u+Q\left(\mathcal{L}^{\prime} L+1\right) u \mid u \in B \cap K\right\} \text {, }
$$

where $Q: v \rightarrow Q v$ is the operator solving the generalized Dirichlet problem for the equation $\mathcal{L}^{\prime} \mathcal{L} Q v+Q v=v\left(\right.$ see Remark 4.3), since $L_{A} K \subset \operatorname{Im} L_{0}$.

2) The cokernel of $L_{B}$ is characterized by the space $B^{+} \cap K^{+}$, which is the projection of the kernel of the conjugate operator.

3) The correctness of the boundary-value problem implies that there is a direct-sum decomposition $A=B \oplus K$ or, which is the same, the direct-sum decomposition $A^{+}=B^{+} \oplus K^{+}$.

Proof. Assertion 1) follows from the orthogonal decomposition $w=P_{A} w+\left(1-P_{A}\right) w$, $\left(1-P_{A}\right) w=Q\left(L^{+} L+1\right) w$. In Assertion 2) the operator $L_{B^{+}}^{+}$is the conjugate of $L_{B}$; we apply assertion 1) to the former. Assertion 3) is an obvious consequence of Propositions 1.2 and 2.12. 
We now consider the process of finding a solution of the boundary-value problem (1.5), which we write in the form: $L u=f \in H^{+}, P_{A} u \in B$. Let $u_{1} \in D(L)$ be any particular solution of the equation $L u=f$. Here we shall not dwell on how to obtain a particular solution, which would require us to develop the technique of solving equations in negative spaces. We project the element $u_{1}$ via the orthogonal projection $P_{A}$ onto the space $A$ : $u_{1}=u_{0}+u_{A}, u_{A}=P_{A} u_{1}, u_{0} \in D\left(L_{0}\right)$. We then project $u_{A}$ along the space $K$ via the projection $P_{B}$ onto the space $B: u_{A}=u_{B}+u_{K}, u_{B} \in B, u_{K} \in K$. We seek the solution of our problem in the form $u=u_{B}+u_{2}, u_{2} \in D\left(L_{0}\right)$. Substituting $u=u_{1}-u_{0}-u_{K}+u_{2}$ in the equation we obtain $L_{0} u_{2}=L_{0} u_{0}+L u_{K}$. But clearly $L u_{K} \in \operatorname{Im} L_{0}$; therefore the element $u_{2}$ is uniquely determined. The projection $P_{A}$, as well as the inverse of the operator $L_{0}$, can be realized by using the methods recommended in Remarks 4.2 and 4.3. We have thus obtained the following result.

Proposition 4.8. The boundary-value problem (1.5) with right-hand side $f \in H^{+}$is solved by carrying out the following sequence of actions:

1) we find a particular solution $u_{1} \in D(L)$ of $L u=f$; this can be done if Vishik's condition (1.2) holds;

2) we find a generalized solution $u_{0}$ of the Dirichlet problem for the equation $L^{+} L u_{0}$ $+u_{0}=\mathcal{L}^{\prime} L u_{1}+u_{1}$ (see Remark 4.3); we find the difference $u_{A}=u_{1}-u_{0}$;

3) we find the projection $u_{B}=P_{B} u_{A}$ of $u_{A}$ onto the subspace $B$ along $K$; this can be done if the problem is correct; we find the difference $u_{K}=u_{A}-u_{B}$;

4) we solve the generalized Dirichlet problem for the equation $\mathcal{L}^{\prime} L_{0} u_{2}=\mathcal{L}^{\prime}\left(L_{0} u_{0}+\right.$ $L u_{K}$ ) (see Remark 4.2), which is correct if Vishik's condition (1.1) holds.

The required solution of the boundary-value problem (1.5) will then be the element $u=u_{B}+u_{2}$.

\section{Some EXAmples}

In this section we carry out calculations which, along with the examples we considered earlier, are used to illustrate some of the ideas discussed above.

5.1. The one-dimensional Laplace operator. Consider the operator

$$
L=\frac{d^{2}}{d x^{2}}, \quad \Omega=I=(0,1) .
$$

In this case the graph norm of the operator is equivalent to the Sobolev norm [32] and $D(L)=H^{2}(I) \subset C^{1}(I), D\left(L_{0}\right)=H_{0}^{2}(I), L^{+}=L$,

$$
\begin{gathered}
\operatorname{ker} L=\left\{C_{1}+C_{2} x \mid C_{1}, C_{2} \in \mathbb{C}\right\}, \\
\operatorname{Im} L_{0}=\left\{u \in L_{2}(I) \mid \int_{I} u(x) d x=\int_{I} x u(x) d x=0\right\} .
\end{gathered}
$$

Green's formula has the form

$$
\int_{I}(L u \bar{v}-u L \bar{v}) d x=-\left.\left(u^{\prime} v-u v^{\prime}\right)\right|_{0} ^{1}
$$

whence

$$
\begin{gathered}
L_{(0)} u=u, \quad L_{(1)} u=-u^{\prime}, \\
A(L)=\left\{u(0), u(1), u^{\prime}(0),-u^{\prime}(1)\right\}=\mathbb{C}^{4}, \\
A(\operatorname{ker} L)=\left\{C_{1}, C_{1}, C_{2},-C_{2}\right\}=C_{1} \vec{a}^{1}+C_{2} \vec{a}^{2} .
\end{gathered}
$$


An arbitrary correct boundary-value problem is given by two vectors $\vec{a}^{3}, \vec{a}^{4}$, which together with the vectors $\vec{a}^{1}, \vec{a}^{2}$ form a basis in $A(L)$. For example, the Dirichlet problem determines the vectors $\vec{a}^{3}=(1,0,0,0)$ and $\vec{a}^{4}=(0,1,0,0)$.

The space $A=A^{+}$is the solution space of the equation $w^{\prime \prime \prime \prime}+w=0$; that is, it is the 4-dimensional subspace of $D(L)$ of functions realizing the boundary values. One of the fundamental solutions $\mathcal{E}(x)=(x+|x|) / 2$ gives a representation of the solution $u$ of the equation $L u=f$ :

$$
u(y)=\int_{0}^{y} f(x)(y-x) d x+u(0)+u^{\prime}(0) y .
$$

5.2. The Laplace operator in a disc. We now consider the Laplace operator as an operation: $\mathcal{L}=\Delta$. Let $L(=\tilde{L})$ be the maximal operator generated by the Laplace operator $\Delta$ and let $\Omega=K=\left\{x \in \mathbb{R}^{2}|| x \mid<1\right\}$ be the disc. The space $D\left(L_{0}\right)$ is the same as the Sobolev space $H_{0}^{2}(K)$, while the space $D(L)=\left\{u \in L_{2}(K) \mid \Delta u \in L_{2}(K)\right\}$ contains $H^{2}(K)$, but does not coincide with it, since, for example, the triple scalar product of functions in $D(L)$ does not necessarily lie in $L_{2}(K)$.

The boundary space $C(L)$ can be identified with the orthogonal complement

$$
A=D(L) \ominus D\left(L_{0}\right)=\operatorname{ker}_{D(L)}\left(\Delta^{2}+1\right) .
$$

The space of associated traces $A(L)$ consists of the $L$-traces $L_{(0)} u=\left.u\right|_{\partial K}=\psi, L_{(1)} u=$ $\left.u_{\nu}^{\prime}\right|_{\partial K}=\chi$. The pair of $L$-traces

$$
\psi=\sum_{n=-\infty}^{\infty} \psi_{n} e^{i n \tau}, \quad \chi=\sum_{n=-\infty}^{\infty} \chi_{n} e^{i n \tau}
$$

belongs to the space $A(\operatorname{ker} \Delta)$ if and only if $\chi_{n}=-n \psi_{n}$, which follows from the representation of a harmonic function in polar coordinates:

$$
u(r, \tau)=\sum_{n=0}^{\infty} r^{n}\left(a_{n} e^{i n \tau}+b_{n} e^{-i n \tau}\right) .
$$

It follows from the same representation that

$$
\sum_{n=1}^{\infty} \frac{1}{n}\left(\left|a_{n}\right|^{2}+\left|b_{n}\right|^{2}\right)<\infty \text { if } u \in L_{2}(K) .
$$

This means that the Fourier series $\psi$ with coefficients $\psi_{n}=a_{n}, \psi_{-n}=b_{n}, n \in \mathbb{N}$, belongs to the space $H^{-1 / 2}(\partial K)$, and the series $\chi \in H^{-3 / 2}(\partial K)$. Hence we see that the space of associated traces $A(L)$ is embedded in the product

$$
H^{(-2)}(\partial K)=H^{-1 / 2}(\partial K) \times H^{-3 / 2}(\partial K),
$$

where both composites of the projections with the embedding $A(L) \rightarrow H^{-1 / 2}(\partial K)$, $A(L) \rightarrow H^{-3 / 2}(\partial K)$ are surjective. In addition, $A(L) \neq H^{(-2)}(\partial K)$, since the pair $(0 ; \chi)$ for $\chi \notin H^{1 / 2}(\partial K), \chi \in H^{-3 / 2}(\partial K)$ does not belong to $A(L)$, since the homogeneous Dirichlet problem for the equation $\Delta u=f \in L_{2}(K)$ demands that the trace $u_{\nu}^{\prime}$ be smooth. This last argument shows that the space of $L$-traces $A(L)$ cannot in general be represented as a direct sum of spaces - images of operators $L_{(j)}$.

We observe that since for regular elliptic operators with constant coefficients of the same order the spaces $D\left(L_{0}\right)$ coincide while the spaces $D(L)$ are separate [45], it follows that their boundary spaces $C(L)$ are separate. In view of the correctness of the Dirichlet problem for such equations it is clear the reason that these spaces are separate is that the kernels of these operators are different. 
For the operator $L=\Delta$ :

$$
\begin{aligned}
\operatorname{ker} L & \left.=\text { (the closure of the linear span of } V=\left\{\exp \left\{\mu\left(x_{1} \pm i x_{2}\right)\right\} \mid \mu \in \mathbb{C}\right\}\right), \\
\operatorname{Im} L_{0} & =\left\{u \in L_{2}(K) \mid \forall \mu \in \mathbb{C}, \int_{K} u(x) \exp \left\{\mu\left(x_{1} \pm i x_{2}\right)\right\} d x=0\right\}
\end{aligned}
$$

An arbitrary correct boundary-value problem (1.5) generates a regular operator $L_{B}$, where $D(L)=\operatorname{ker} L \oplus D\left(L_{B}\right)$ and $C(L)=C(\operatorname{ker} L) \oplus \Gamma D\left(L_{B}\right)$, in particular, for the Dirichlet problem $B=\left\{u \in L_{2}(K)\left|L_{(0)} u=u\right|_{\partial K}=0\right\}$, as is known, and $D\left(L_{B}\right)=$ $H^{2}(K) \cap H_{0}^{1}(K)$. Concerning the connection between Vishik's theorem on the correctness of boundary-value problems for elliptic equations, see below in $\S 6$.

5.3. The wave operator in a disc. We consider the wave operator

$$
L=\square=\frac{\partial^{2}}{\partial x_{1} \partial x_{2}}
$$

in the unit $\operatorname{disc} \Omega=K=\left\{x \in \mathbb{R}^{2}|| x \mid<1\right\}$.

The space $D\left(L_{0}\right)$ contains the Sobolev space $H_{0}^{2}(K)$, which is contained in the Sobolev space $H_{0}^{1}(K)$ since the wave operator is of principal type (see $\S 1.2$ ) and, as Proposition 3.1 shows, $D\left(L_{0}\right) \cap H^{2}(K)=H_{0}^{2}(K)$, since (3.2) holds. The space $D(L)=\left\{u \in L_{2}(K) \mid\right.$ $\left.\square u \in L_{2}(K)\right\}$ contains $H^{2}(K)$ but does not coincide with it since, for example, the second partial derivatives of a function in $D(L)$ do not necessarily belong to $L_{2}(K)$.

The boundary space is identified with the orthogonal complement

$$
C(L)=A=D(L) \ominus D\left(L_{0}\right)=\operatorname{ker}_{D(L)}\left(\square^{2}+1\right) .
$$

The space of associated traces $A(L)$ consists of the $L$-traces

$$
\begin{aligned}
& L_{(0)} u=\left.\lambda(\nu(x)) u\right|_{\partial K}=\left.x_{1} x_{2} u\right|_{\partial K} \in H^{-1 / 2}(\partial \Omega), \quad \lambda=x_{1} x_{2}, \\
& L_{(1)} u=-\lambda(x) u_{\nu}^{\prime}+\lambda_{\tau}^{\prime} u_{\tau}^{\prime}+\left.\frac{1}{2} \lambda_{\tau \tau}^{\prime \prime} u\right|_{\partial K} \in H^{-3 / 2}(\partial \Omega) \quad \text { (see Example 2.1). }
\end{aligned}
$$

For the operator $L=\square$ :

ker $L=$ (the closure of the linear span $\left.V=\left\{e^{\mu x_{j}} \mid \mu \in \mathbb{C}, j=1,2\right\}\right)$,

$$
\operatorname{Im} L_{0}=\left\{u \in L_{2}(K) \mid \forall \mu \in \mathbb{C}, j=1,2, \int_{K} u(x) e^{\mu x_{j}} d x=0\right\} .
$$

An arbitrary correct boundary-value problem (1.5) generates a regular operator $L_{B}$ with $D(L)=\operatorname{ker} L \oplus D\left(L_{B}\right)$ and $C(L)=C(\operatorname{ker} L) \oplus \Gamma D\left(L_{B}\right)$. We note that an example of a correct boundary-value problem was given in $\S 1.3$.

\section{Coordinatization of the Spaces $A$ And $A^{+}$}

In this section we discuss possible directions for further study of general boundaryvalue problems for general differential equations.

We have already noted that if a boundary-value problem is correct, then one has the direct-sum expansion $A=B \oplus K$ or, which is the same, the direct-sum expansion $A^{+}=B^{+} \oplus K^{+}$; and in order to solve boundary-value problem (1.5) one needs to know how to find the projection of an element $u \in A$ onto the subspace $B$ along $K$. In order to be able to pose and solve the problem of making this decomposition into a direct sum one needs to invoke some kind of additional structures or classes of operators. From our point of view, the natural approach here is the coordinatization of the spaces $A$ and $A^{+}$, that is, the introduction of "functional" coordinates, primarily in relation to traces. We discuss the possibilities for solving this problem. 
The boundary-value problem (1.5), written in the space $A$ as $u \in B \subset A$, can, as in Proposition 2.13 (formula (2.19)), be written in the form

$$
B_{i}^{+0} \tilde{L}_{(0)} u+B_{i}^{+1} \tilde{L}_{(1)} u+\cdots+B_{i}^{+l-1} \tilde{L}_{(l-1)} u=0, \quad i=1, \ldots, l,
$$

where

$$
u \in A, B_{i}^{+k}: \tilde{H}_{\partial}^{+-k-1 / 2} \rightarrow \tilde{H}_{\partial}^{+-i-1 / 2}
$$

are certain continuous operators. We can also write the spaces $K$ and $K^{+}$in the same form. However, making use of these formulae is awkward in view of the following. By carrying out the idea of coordinatization we could, by identifying the space $A$ with the space $A(L) \subset H^{+(-l)}$ of $L$-traces, obtain the coordinates of the element $u \in A$ in the form of a tuple of its $L$-traces $\left(L_{(0)} u, \ldots, L_{(l-1)} u\right)$, but we cannot assume here that a coordinate $\left(0, \ldots, 0, L_{(k)} u, 0, \ldots, 0\right)$ taken individually belongs to the space $A(L)$. Indeed an example where this happens is the Laplace operator in a disc (see §5.2).

A classical example giving the solution to the problem of coordinates in the boundary space is the introduction of boundary operators $\gamma_{1}: D(L) \rightarrow H^{-1 / 2}(\partial \Omega), \gamma_{2}: D(L) \rightarrow$ $H^{1 / 2}(\partial \Omega)$, acting according to the rules:

$$
\gamma_{1} u=\left.u\right|_{\partial \Omega}, \gamma_{2} u=\left.u_{\nu}^{\prime}\right|_{\partial \Omega}-P \gamma_{1} u
$$

where $P \gamma_{1} u=\left.v_{\nu}^{\prime}\right|_{\partial \Omega}, L v=0,\left.\gamma_{1} v\right|_{\partial \Omega}=\left.\gamma_{1} u\right|_{\partial \Omega}$ (this is due to Vishik [18 for the case of a uniformly elliptic second-order operation in a bounded domain with smooth boundary). Here we use in explicit form, firstly, the correctness of the Dirichlet problem, which (since (1.1) and (1.2) hold) enables us to identify the kernel $\operatorname{ker} L^{+}(=\operatorname{ker} L)$ with the range of the operator $\gamma_{1}$, in view of the decomposition $D\left(L^{+}\right)=\operatorname{ker} L^{+} \oplus$ $D\left(L_{B^{+}}^{+}\right), B=B^{+}=\left\{\gamma_{1} u=0\right\}$; secondly, the correctness of the problem, $L u=f$, $\gamma_{2} u=0$, enabling us to identify the kernel $\operatorname{ker} L$ with the range of the operator $\gamma_{2}$; and thirdly, the possibility of describing the range of the operator $\gamma_{2}$ as the space $H^{1 / 2}(\partial \Omega)$. In accordance with Proposition 1.2 , we may now decompose the boundary space into a direct sum: $C(L)(\approx A(L))=\operatorname{ker} L^{+} \oplus \operatorname{ker} L$ (we have $A=K \oplus L_{A^{+}}^{+} K^{+}$) and $C(L) \approx H^{-1 / 2}(\partial \Omega) \oplus H^{1 / 2}(\partial \Omega)$. Also, in accordance with Proposition 1.3, we are now in a position to describe all correct boundary-value problems via the Vishik operator $V: \operatorname{ker} L^{+} \rightarrow \operatorname{ker} L$, as was done in [18.

Approaching from the other direction, for each $k=0, \ldots, l-1$ we introduce the subspace

$$
H_{0 k}^{l}=\left\{w \in H^{l} \mid \forall j \neq k, \gamma_{j} w\left(=\left.\partial_{\nu}^{j} w\right|_{\partial \Omega}\right)=0\right\}=H_{0}^{l} \oplus H_{\partial}^{l-k-1 / 2},
$$

its closure $T_{k}:=\operatorname{Clos}_{D(L)} H_{0 k}^{l}$ and the projection $\tilde{T}_{k}:=P_{A} T_{k}=A \cap T_{k}$; similarly for the "plus" spaces we introduce the subspace

$$
H_{0 k}^{+l}=H_{0}^{+l} \oplus H_{\partial}^{+l-k-1 / 2}
$$

its closure $T_{k}^{+}:=\operatorname{Clos}_{D\left(L^{+}\right)} H_{0 k}^{+l}$, and the projection $\tilde{T}_{k}^{+}:=A^{+} \cap T_{k}^{+}$. There are now two possibilities.

The first possibility is that we can carry out, so to speak, the "orthogonalization" of the spaces $\tilde{T}_{k}$ and $\tilde{T}_{k}^{+}$. Namely, we consider the closures of the successive sums

$$
\begin{aligned}
& D\left(L_{1}\right)=\operatorname{Clos}_{D(L)}\left(H_{0}^{l}+H_{\partial}^{l-1 / 2}\right)=\operatorname{Clos}_{D(L)}\left(D\left(L_{0}\right)+T_{0}\right), \\
& D\left(L_{2}\right)=\operatorname{Clos}_{D(L)}\left(H_{0}^{l}+H_{\partial}^{l-1 / 2}+H_{\partial}^{l-3 / 2}\right)=\operatorname{Clos}_{D(L)}\left(D\left(L_{0}\right)+T_{0}+T_{1}\right), \\
& D\left(L_{l-1}\right)=\operatorname{Clos}_{D(L)}\left(H_{0}^{l}+H_{\partial}^{l-1 / 2}+\cdots+H_{\partial}^{3 / 2}\right)=\operatorname{Clos}_{D(L)}\left(D\left(L_{0}\right)+T_{0}+\cdots+T_{l-2}\right), \\
& D\left(L_{l}\right)=D(\tilde{L}) .
\end{aligned}
$$


We then consider the system of orthogonal differences

$$
A_{1}=D\left(L_{1}\right) \ominus D\left(L_{0}\right), A_{2}=D\left(L_{2}\right) \ominus D\left(L_{1}\right), \ldots, A_{l-1}=D(\tilde{L}) \ominus D\left(L_{l-2}\right) .
$$

Suppose that the following conditions hold:

$$
\text { for } k=0, \ldots, l-1 \text {, the space } \operatorname{Im} L_{k} \text { is closed in } H^{+} \text {, }
$$

which hold, in particular, when (4.2) is satisfied. Consider the analogous properties for the operator $L_{k}^{+}$, which hold when (4.1) is satisfied. Then we obtain orthogonal expansions of the type of part 1) of Proposition 1.2: $D\left(L_{j}\right)=D\left(L_{j-1}\right) \oplus K_{j} \oplus L^{+} K_{j}^{+}$. We then consider Green's formulae, write the boundary-value problem in the form of a linear system with operator-valued coefficients, and so on.

The second possibility is that we can immediately isolate the class of operations for which the two sums consisting of the various spaces $\tilde{T}_{j}$ are nonintersecting and the sum of the spaces $\tilde{T}_{k}$ coincides with the space $A$. For this, we clearly require that the angles between $\tilde{T}_{k}$ and $D\left(L_{k-1}\right)$ be positive. This approach is used in the same way as that enabling one to define the notion of the ordinary trace of an element in the space $A$ as the projection of this element onto the $\tilde{T}_{k}$ "axis" and carry over the tangent operators and formulae of type (0.3) to the decomposition of the spaces $A$ and $A^{+}$.

In both cases we find ourselves in the area of the linear algebra of Hilbert modules over a noncommutative ring of operators. This, in our view, is a subject for further investigation.

\section{Comments}

We recall that Hörmander [45] defined the Cauchy space as the quotient $G(L) / G\left(L_{0}\right)$, where

$$
G(L)=\left\{(u, L u) \in D(L) \times H^{+} \mid u \in D(L)\right\}
$$

is the graph of $L$, and $G\left(L_{0}\right)$ is the graph of $L_{0}$. The definition of the Cauchy space is equivalent to the definition of the boundary space introduced above, making the identifications $G(L)=D(L), G\left(L_{0}\right)=D\left(L_{0}\right)$. Note that the term Cauchy space was not established or applied to the quotient space $D(L) / D\left(L_{0}\right)$. In the literature (for example, [19, 17, 27]) the term "space of boundary values" is used, but in this connection it is supposed that in the structure "space of boundary-value problems" there also occur operators entering in the so-called abstract Green's formula and Green's formula itself; therefore the application of this term to denote the quotient space $D(L) / D\left(L_{0}\right)$ without these operators is unjustified. Another thing against the term "Cauchy space" is that (as was clear from the above) the space $C(L)$ does not consist of Cauchy data, by which one might mean the family of traces $\left.u\right|_{\partial \Omega},\left.u_{\nu}^{\prime}\right|_{\partial \Omega}$, and does not even consist of $L$-traces (see $\S 2$ ); in other words, the term "Cauchy space" is, in our view, misleading. Therefore we use the neutral term "boundary space". The Green's formula, in which expressions for the $L$-traces of a smooth function with general differential operator of higher order appeared for the first time, was described in Routberg's papers [38, 39]. Condition (2.15) in the form of the divisibility of the Fourier transform from $\left[L, \theta_{\Omega}\right] u=L\left(\theta_{\Omega} u\right)-\theta_{\Omega} L u$ by the symbol of the operator $L$ was first published by Roitberg [40. for a half-space and simultaneously and independently by this author [15] for the disc. We note that the concept of the $L$-trace of a function in $D(\tilde{L})$ appeared in the available literature in the works of this author [5, 11, although we used the expressions for the $L$-traces even earlier in calculations relating to the study of specific nonclassical and general boundary-value problems [4, 8, 6, 7, 15, 16. We further note that these same expressions for $L$-traces of smooth functions were used long before that and were derived whenever the need arose for some or other differential operator in Green's formula; for the case of general linear 
differential operations, as we have remarked, these expressions can be found in the papers of Roitberg [38, 39. We also emphasize that in the same papers by Routberg, in fact, the $L$-traces of a nonsmooth solution of the equation $L u=f$ in negative Sobolev spaces are implicitly used, in one of the stages of the proof of the existence of ordinary traces of the solution in a negative space in a neighbourhood of a noncharacteristic point of the boundary. These and other properties of the space $A=D(L) \ominus D\left(L_{0}\right)$ were used in the theory of selfadjoint extensions of a symmetric operator (see, for example, 22]), and in the general case encountered in articles by Lyantse (with finite deficiency indices) and Lyantse and Storozh [31] (with arbitrary deficiency indices).

\section{REFERENCES}

[1] M.S. Agranovich, On partial differential equations with constant coefficients, Uspekhi Mat. Nauk 16 (1961), no. 2, 27-93. (Russian) MR0133597

[2] Yu.M. Berezanskiı̌, Expansions in eigenfunctions of selfadjoint operators, Naukova Dumka, Kiev, 1965. (Russian) MR0222719

[3] N. Bourbaki, Topological vector spaces, Springer-Verlag, Berlin, 1987. MR0910295

[4] V.P. Burskiı̌, Harmonic analysis in boundary-value problems for partial differential equations with constant coefficients, Dokl. Akad. Nauk Ukrain. SSR Ser. A 1986, no. 12, 7-10. (Russian) MR 0842785

[5] V.P. Burskiü, Boundary properties of $L_{2}$-solutions of linear differential equations and the equationdomain duality, Dokl. Akad. Nauk SSSR 309 (1989), no. 5, 1036-1039; English transl. in Soviet Math. Dokl. 40 (1990), no. 3, 592-595. MR.1037114

[6] V.P. Burskilu, Uniqueness of the solution of the Dirichlet problem in a ball for the wave equation, Differ. Uravn. 24 (1988), no. 6, 1038-1039. (Russian) MR0953854

[7] V.P. Burskiı̌, Remarks on the Dirichlet problem for an ultrahyperbolic equation in a ball and integral geometry on the sphere, Uspekhi Mat. Nauk 43 (1988), no. 5, 181-182; English transl. in Russian Math. Surveys 43 (1988), no. 5, 215-216. MR0971474

[8] V.P. Burskiŭ, Boundary value problems for a second-order hyperbolic equation in a disk, Izv. Vyssh. Uchebn. Zaved. Mat. 1987, no. 2, 22-29; English transl. in Soviet Math. (Iz. VUZ) 31 (1987), no. 2, 34-47. MR0889192

[9] V.P. Burskiı̌, Methods of investigating boundary-value problems for general differential equations, Naukova Dumka, Kiev, 2002. (Russian)

[10] V.P. Burskiı̌, Generalized solutions of boundary value problems for differential equations of general type, Uspekhi Mat. Nauk 53 (1998), no. 4, 215-216; English transl. in Russian Math. Surveys 53 (1988), no. 4, 864-865. MR.1668066

[11] V.P. Burskiı̌, A commutative diagram connected with a differential operator in a domain, Ukrain. Mat. Zh. 43 (1991), no. 12, 1703-1709; English transl. in Ukrainian Math. J. 43 (1991), no. 12, 1588-1594. MR,1172313

[12] V.P. Burskiı, On the uniqueness of the solution of some boundary value problems for differential equations in a domain with an algebraic boundary, Ukrain. Mat. Zh. 45 (1993), no. 7, 898-906; English transl. in Ukrainian Math. J. 45 (1993), no. 7, 993-1003. MR1260648

[13] V.P. Burskiı̌, Boundary-value problems for an elliptic equation with complex coefficients and a moment problem, Ukrain. Mat. Zh. 45 (1993), no. 11, 1476-1483; English transl. in Ukrainian Math. J. 45 (1993), no. 11, 1659-1668. MR1307364

[14] V.P. Burskiǔ, Solutions of the Dirichlet problem in a disk, Ukrain. Mat. Zh. 44 (1992), no. 10, 1307-1313; English transl. in Ukrainian Math. J. 44 (1992), no. 10, 1197-1203. MR.1201128

[15] V.P. Burskiı, On the kernel of a differential operator with constant lower-order coefficients in a disk, Manuscript deposited in VINITI, no. 3796-82 Dep. (Russian)

[16] V.P. Burskiॅ, Theorems on traces of the solution of the string vibration equation in the disk, Akad. Nauk Ukrain. SSR Inst. Mat. Preprint 1985, no. 23.(Russian) MR0830507

[17] L.I. Vaŭnerman, Extensions of closed operators in Hilbert space, Mat. Zametki 28 (1980), no. 6, 833-842. English transl., Math. Notes 28 (1980), no. 5-6, 871-875. MR0603218

[18] M.I. Vishik, On general boundary problems for elliptic differential equations, Tr. Moskov. Mat. Obshch. 1 (1952), 187-246. (Russian) MR0051404(14:473d)

[19] V.I. Gorbachuk and M.L. Gorbachuk, Boundary value problems for operator differential equations, Naukova Dumka, Kiev, 1984. (Russian) MR0776604 
[20] V.I. Gorbachuk, M.L. Gorbachuk and A.N. Kochubeř, The theory of extensions of symmetric operators, and boundary value problems for differential equations, Ukrain. Mat. Zh. 41 (1989), no. 10, 1299-1313; English transl. in Ukrainian Math. J. 41 (1989), no. 10, 1117-1129. MR.1034669

[21] M.L. Gorbachuk, Selfadjoint boundary value problems for a second-order differential equation with unbounded operator coefficients, Funkt. Anal. 5 (1971), no. 1, 10-21. English transl., Functional Anal. Appl. 5 (1971), 9-18. MR0283624

[22] N. Dunford and J.T. Schwartz, Linear operators, II. Spectral theory, Wiley, New York, 1988; Russian transl., Mir, Moscow, 1966. MR1009163

[23] A.A. Dezin, General questions of the theory of boundary value problems, Nauka, Moscow, 1980. MR0596223

[24] Yu.V. Egorov and M.A. Shubin, Linear partial differential equations. Foundations of the classical theory, Itogi Nauchn. i Tekhn. Inform., Moscow, 1988. English transl., Partial Differential Equations I, Encyclopedia of Mathematical Sciences, 30, Springer-Verlag, 1992. MR.1141629

[25] M.G. Kreĭn, Theory of selfadjoint extensions of semibounded Hermitian operators and its applications, Mat. Sb. 20 (1947), no. 3, 431-495. (Russian) MR0024574

[26] S.G. Krĕn, Linear equations in a Banach space, Nauka, Moscow, 1971; English transl., Birkhäuser, Boston, Mass, 1972. MR0374949

[27] A.N. Kochuber, Extensions of symmetric operators and of symmetric binary relations, Mat. Zametki 17 (1975), 41-48.English transl., Math. Notes 17(1975), no.1, 25-28. MR0365218

[28] L.P. Kuptsov, The mean value property and the maximum principle for second order parabolic equations, Dokl. Akad. Nauk SSSR 242 (1978), no. 3, 529-532. English transl., Soviet Math. Dokl. 19 (1978), no. 5, 1140-1144. MR.0507137

[29] O.A. Ladyzhenskaya and N.N. Ural'tseva, Linear and quasilinear equations of elliptic type, Nauka, Moscow, 1973; English transl., Academic Press, New York-London, 1968. MR0509265 (58:29032) MR 0244627

[30] J.-M. Lions and E. Magenes, Problèmes aux limites non homogènes et applications, Dunod, Paris, 1968. MR0247243.

[31] V.E. Lyantse and O.G. Storozh, Methods of the theory of unbounded operators, Naukova Dumka, Kiev, 1983. (Russian) MR0757535

[32] V.G. Maz'ya, Sobolev spaces, Leningrad Univ., Leningrad, 1985; English transl., Springer-Verlag, Berlin, 1985. MR0817985

[33] A.Kh. Mamyan, The construction of solvable extensions in a parallelepiped for linear differential operators with constant coefficients, Differ. Uravn. 6 (1970), 358-370. (Russian) MR0279426

[34] M.A. Naŭmark, Linear differential operators, Nauka, Moscow, 1969; English transl., Ungar, New York, 1968. MR 0262880

[35] V.P. Palamodov, Linear differential operators with constant coefficients, Nauka, Moscow, 1967; English transl., Springer-Verlag, Berlin, 1970. MR.0264197

[36] B.P. Paneah, On general systems of differential equations with constant coefficients, Dokl. Akad. Nauk SSSR 138 (1961), no. 2, 297-300. (Russian) MR0126606

[37] B.I. Ptashnik, Ill-posed boundary value problems for partial differential equations, Naukova Dumka, Kiev, 1984. (Russian) MR0772024

[38] Ya.A. Roltberg, The existence of limit values of generalized solutions of elliptic equations on the boundary of a domain, Sibirsk. Mat. Zh. 20 (1979), no. 2, 386-396; English transl. in Siberian Math. J. 20 (1979), no. 2, 276-283. MR0530503

[39] Ya.A. Routtberg, On the boundary values of generalized solutions of systems that are elliptic in the sense of Douglis and Nirenberg, Sibirsk. Mat. Zh. 18 (1977), no. 4, 846-860; English transl. in Siberian Math. J. 18 (1977), no. 4, 600-610. MR0486967

[40] Ya.A. Roĭtberg and V.A. Serdyuk, Elliptic problems with a parameter in $L_{2}$-spaces of generalized functions for general systems of equations, Akad. Nauk Ukrain. SSR Inst. Mat. Preprint 1982, no. 30. (Russian) MR0692462 (84b:35041)

[41] F.S. Rofe-Beketov, Selfadjoint extensions of differential operators in a space of vector-valued functions, Funktsional. Anal. i Prilozhen. 8 (1969), 3-24. (Russian) MR0281055

[42] I.V. Skrypnik, Non-linear elliptic differential equations, Naukova Dumka, Kiev, 1973. (Russian) MR 0435590

[43] L. Hörmander, The analysis of linear partial differential operators I, Springer-Verlag, Berlin, 1985. MR0717035 (85g:35002a)

[44] L. Hörmander, Differential operators of principal type, Math. Ann. 140 (1960), 124, 146. MR0130574 (24:A434)

[45] L. Hörmander, The analysis of linear partial differential operators I, 2nd ed., Springer-Verlag, Berlin, 1990. MR:1065136(91m:35001b) 
[46] L. Hörmander, The analysis of linear partial differential operators II, Springer-Verlag, Berlin, 1983. MR705278 (85g:35002b)

[47] V.P. Burskir. On the well-posedness of boundary value problems for some class of general PDEs in a generalized setting, Funct. Differ. Eq. 8 (2001), no. 1-2, 89-100. MR.1949991

[48] J. Calkin, Abstract symmetric boundary conditions, Trans. Amer. Math. Soc. 45 (1939), no. 3, 369-442. MR 1501997

[49] A. Douglis and L. Nirenberg, Interior estimates for elliptic systems of partial differential equations, Comm. Pure and Appl. Math. 8 (1955), no. 4, 503-538. MR0075417

[50] H. Gajewski, K. Groger and K. Zacharias, Nichtlineare Operatorgleichungen und Operatordifferentialgleichungen. Academie-Verlag, Berlin, 1974. MR0636412

[51] G. Grubb, A characterisation on the non-local boundary value problems associated with an elliptic operator, Ann. Sc. Norm. Sup. Pisa 22 (1968), 425-523. MR0239269

[52] G. Gudmundsdottir, Global properties of differential operators of constant strength, Ark. Mat. 15 (1977), 169-198. MR0509109

[53] V.A. Derkach and M. M. Malamud, Generalized resolvents and boundary-value problems for Hermitian operators with gaps, J. Funct. Anal. 95 (1991), 1-96. MR1087947

[54] M.M. Malamud and V.I. Mogilevskii, Krein type formula for canonical resolvents of dual pairs of linear relations, Methods Funct. Anal. Topology 8 (2002), no. 4, 72-100. MR1942823

[55] L. Hörmander, Definitions of maximal operators, Arkiv för Mat. 3 (1958), no. 46, 501-504. MR0106333(21:5067)

[56] B. Fuglede, A priori inequalities connected with systems of partial differential equations, Acta Math. 105 (1961), 177-195. MR0140818

Institute of Applied Mathematics and Mechanics, National Academy of Sciences of Ukraine E-mail address: v30@dn.farlep.net 